\title{
What's in a crowd? Analysis of face-to-face behavioral networks
}

\author{
Lorenzo Isella, Ciro Cattuto, and Wouter Van den Broeck \\ Complex Networks and Systems Group, Institute for Scientific Interchange (ISI) Foundation, Turin, Italy \\ Juliette Stehlé and Alain Barrat \\ Centre de Physique Théorique, CNRS UMR 6207, Marseille, France \\ Jean-François Pinton \\ Laboratoire de Physique de l'ENS Lyon, CNRS UMR 5672, Lyon, France
}

\begin{abstract}
The availability of new data sources on human mobility is opening new avenues for investigating the interplay of social networks, human mobility and dynamical processes such as epidemic spreading. Here we analyze data on the time-resolved face-to-face proximity of individuals in large-scale real-world scenarios. We compare two settings with very different properties, a scientific conference and a long-running museum exhibition. We track the behavioral networks of face-to-face proximity, and characterize them from both a static and a dynamic point of view, exposing differences and similarities. We use our data to investigate the dynamics of a susceptible-infected model for epidemic spreading that unfolds on the dynamical networks of human proximity. The spreading patterns are markedly different for the conference and the museum case, and they are strongly impacted by the causal structure of the network data. A deeper study of the spreading paths shows that the mere knowledge of static aggregated networks would lead to erroneous conclusions about the transmission paths on the dynamical networks.
\end{abstract}

\section{INTRODUCTION}

Access to large data sets on human activities and interactions has long been limited by the difficulty and cost of gathering such information. Recently, the ever increasing availability of digital traces of human actions is widely enabling the representation and the analysis of massive amounts of information on human behavior. The representation of this information in terms of complex networks [1 8] has led to many research efforts because of the naturally interlinked nature of these new data sources.

Tracing human behavior in a variety of contexts has become possible at very different spatial and temporal scales: from mobility of individuals inside a city 9 and between cities 10, to mobility and transportation in an entire country [11, all the way to planetary-scale travel [12, 13. Mobile devices such as cell phones make it possible to investigate mobility patterns and their predictability [14, 15]. On-line interactions occurring between individuals can be monitored by logging instant messaging or email exchange [16 21. Recent technological advances further support mining real-world interactions by means of mobile devices and wearable sensors, opening up new avenues for gathering data on human and social interactions. Bluetooth and Wifi technologies give access to proximity patterns [22 26], and even face-to-face presence can be resolved with high spatial and temporal resolution [27 30. The combination of these technological advances and of heterogeneous data sources allow researchers to gather longitudinal data that have been traditionally scarce in social network analysis 31, 32. A dynamical perspective on interaction networks paves the way to investigating interesting problems such as the interplay of the network dynamics with dynamical processes taking place on these networks.

In this paper, we capitalize on recent efforts 27+30 that made possible to mine behavioral networks of face-to-face interactions between individuals, in a variety of real-world settings and in a time-resolved fashion. We present an in-depth analysis of the data we collected at two widely different events. The first event was the INFECTIOUS exhibition 33 held at the Science Gallery in Dublin, Ireland, from April $17^{\text {th }}$ to July $17^{\text {th }}$, 2009. The second event was the ACM Hypertext 2009 conference [34] hosted by the Institute for Scientific Interchange Foundation in Turin, Italy, from June $29^{\text {th }}$ to July $1^{\text {st }}$, 2009. In the following, we will refer to these events as SG and HT09, respectively. Intuitively, interactions among conference participants differ from interactions among museum visitors, and the concerned individuals have very different goals in both settings. The study of the corresponding networks of proximity and interactions, both static and dynamic, reveals indeed strong differences but also interesting similarities. We take advantage of the availability of time-resolved data to show how dynamical processes that can unfold on the close proximity network - such as the propagation of a piece of information or the spreading of an infectious agent - unfold in very different ways in the investigated settings. In the epidemiological literature, traditionally, processes of this kind have been studied using either aggregated data or under assumptions of stationarity for the interaction networks: here we leverage the time-resolved nature of our data to assess the role of network dynamics 
on the outcome of spreading processes. At a more fundamental level, simulating simple spreading processes over the recorded interaction networks allows us to expose several properties of their dynamical structure as well as to probe their causal structure.

The paper is organized as follows: first, we briefly describe the data collection platform and our data sets in Section II in Section III we discuss the salient features of the networks of interactions aggregated on time windows of one day. These networks are static objects, carrying only information about the cumulative time that - daily - each pair of individuals has spent in face-to-face proximity. Section IV analyzes the dynamical properties of face-to-face interactions between conference participants and museum visitors. Section $\mathrm{V}$ further characterizes the aggregated network structures by investigating the effect of incremental link removal. Finally, Section VI investigates the role played by causality in information spreading along the proximity network, and Section VII concludes the paper and defines a number of open questions.

\section{DATA}

The data collection infrastructure uses active Radio-Frequency Identification Devices (RFID) embedded in conference badges to mine face-to-face proximity relations of persons wearing the badges. RFID devices exchange ultra-low power radio packets in a peer-to-peer fashion, as described in Refs. 27. 30]. Exchange of radio packets between badges is only possible when two persons are at close range (1 to $1.5 \mathrm{~m}$ ) and facing each other, as the human body acts as a RF shield at the carrier frequency used for communication. The operating parameters of the devices are programmed so that the face-to-face proximity of two individuals wearing the RFID tags can be assessed with a probability in excess of $99 \%$ over an interval of 20 seconds, which is a fine enough time scale to resolve human mobility and proximity at social gatherings. False positives are exceedingly unlikely, as the ultra-low power radio packets used for proximity sensing cannot propagate farther than 1.5-2m, and a sustained excess of packets is needed in order to signal a proximity event. When a relation of face-to-face proximity (or "contact", as we will refer to it in the following) is detected, the RFID devices report this information to receivers installed in the environment (RFID readers). The readers are connected to a central computer system by means of a Local Area Network. Once a contact has been established, it is considered ongoing as long as the involved devices continue to exchange at least one radio packet for every subsequent interval of 20 seconds. Conversely, a contact is considered terminated if an interval of 20 seconds elapses with no packets exchanged. For a detailed description of the sensing platform and some of its deployments, see Refs. 27 30].

The deployments at the Science Gallery in Dublin [33] and at the HT09 conference in Turin [34] involved vastly different numbers of individuals and stretched along different time scales. The former lasted for about three months and recorded the interactions of more than 14,000 visitors (more than 230, 000 face-to-face contacts recorded), whereas the latter took place over the course of three days and involved about 100 conference participants (about 10,000 contacts). Behaviors are also very different: in a museum, visitors typically spend a limited amount of time on site, well below the maximum duration permitted by the museum opening hours, they are not likely to return, and they follow a rather pre-defined path, touching different locations that host the exhibits. In a conference setting, on the other hand, most attendees stay on-site for the entire duration of the conference (a few days), and move at will between different areas such as conference room, areas for coffee breaks and so on. The coverage of the community was different in both settings. At the Science Gallery, visitors were equipped with a RFID tag upon entering the venue, as part of an interactive exhibit, and therefore almost the totality of them were tracked. On the other hand, at HT09, about $75 \%$ of the participants volunteered to being tracked. This sampling may introduce some biases in the results. Sampling issues are also commonly encountered in the study of static complex networks [35. 38. Reference [28] has shown that for a broad variety of real-world deployments of the RFID proximity-sensing platform used in this study, the behavior of the statistical distributions of quantities such as contact durations is not altered by unbiased sampling of individuals. On the other hand, we cannot completely rule out that a systematic bias is introduced by the selection of volunteers, if volunteers and non-volunteers have different behavioral patterns. Accurately checking this point would require monitoring an independent data source for face-to-face contacts, and because of scalability issues this would be feasible only for small control groups. Issues regarding the effect of missing data and incomplete sampling on the properties of dynamical processes unfolding on the networks also deserve attention and will be the subject of future investigations.

\section{THE STATIC INTERACTION NETWORK}

We start by analyzing aggregated networks of interaction obtained by aggregating the raw proximity data over one day. This aggregation yields a social graph where nodes represent individuals, and an edge is drawn between two nodes if at least one contact was detected between those nodes during the interval of aggregation. Therefore, every 


\section{HT09: June $30^{\text {th }}$}

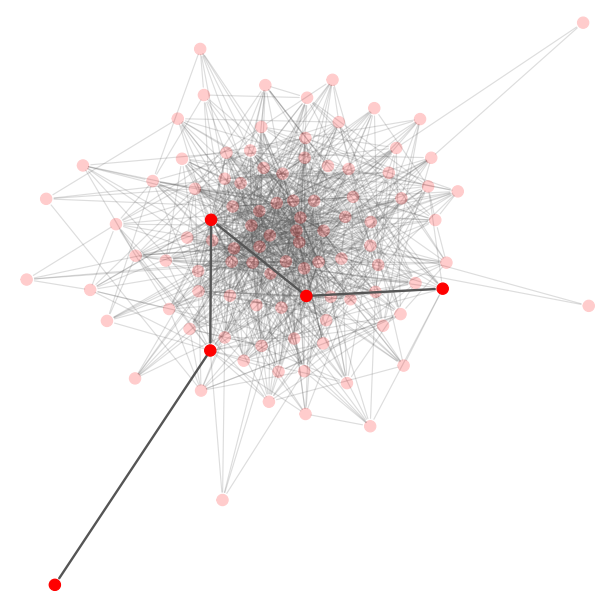

SG: May $19^{\text {th }}$

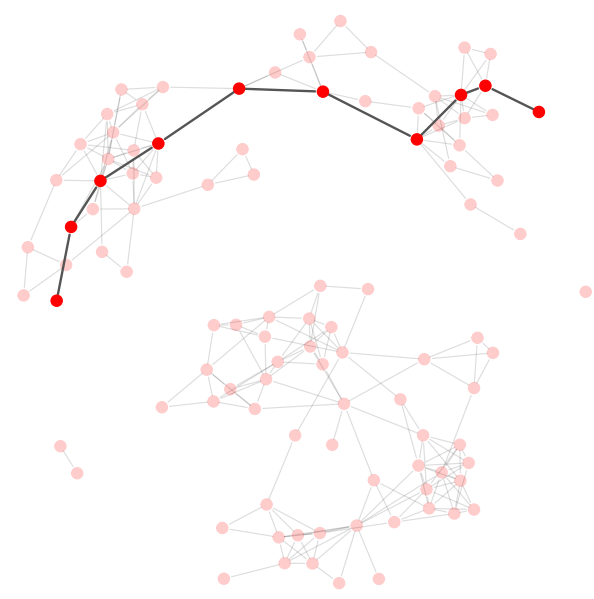

SG: July $14^{\text {th }}$

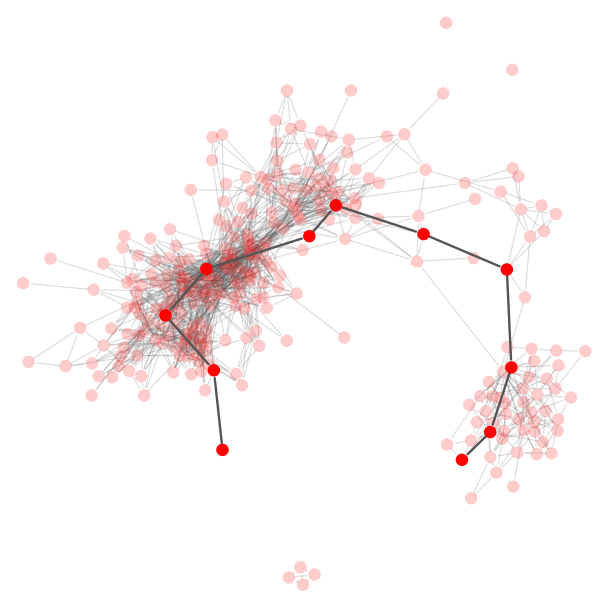

SG: May $20^{\text {th }}$

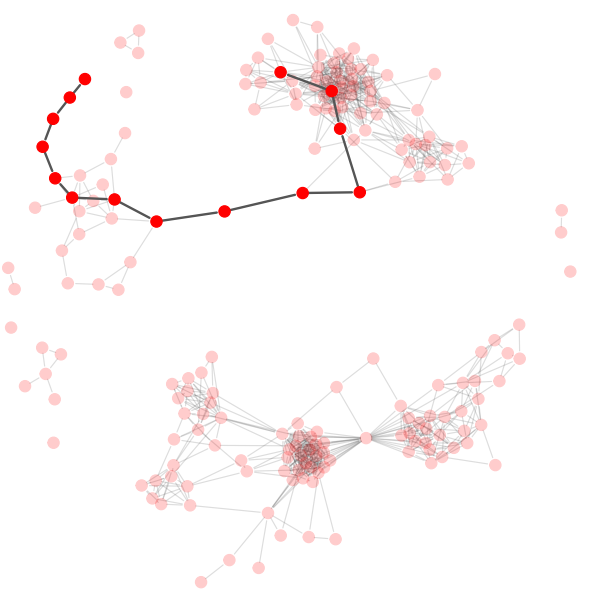

FIG. 1: Daily aggregated networks in the HT09 and SG deployments. Nodes represent individuals and edges are drawn between nodes if at least one contact event was detected during the aggregation interval. Clockwise from top: aggregated network for one day of the HT09 conference, and for three representative days at the SG deployment. In each case, the network diameter is highlighted. All the network visualizations in this study were produced using the igraph library [56].

edge is naturally weighted by the total duration of the contact events that occurred between the tags involved, i.e., by the total time during which the corresponding individuals have been in face-to-face proximity.

The choice of daily time windows seems quite natural in our settings. It would represent, for instance, a typical time scale for a description of articulated social networks based on surveys, in which each participant would (ideally) declare who s/he has encountered during the course of the day. Such a choice for the duration of the time-window, albeit natural, is by no means unique [26. For instance, it is possible to aggregate the data over longer periods of time (weeks or months) to investigate the stationarity of the collected data [28]. Shorter aggregation times of the order of a few minutes are also useful, for instance, to resolve circadian activity patterns at the venue under investigation.

Figure 1 displays the aggregated contact networks for June $30^{\text {th }}$ at the HT09 conference (top left), and for three representative days for the SG museum deployment. Despite the large variation in the number of daily museum visitors, ranging from about 60 to 400, the chosen days illustrate many features of the SG aggregated networks, in 


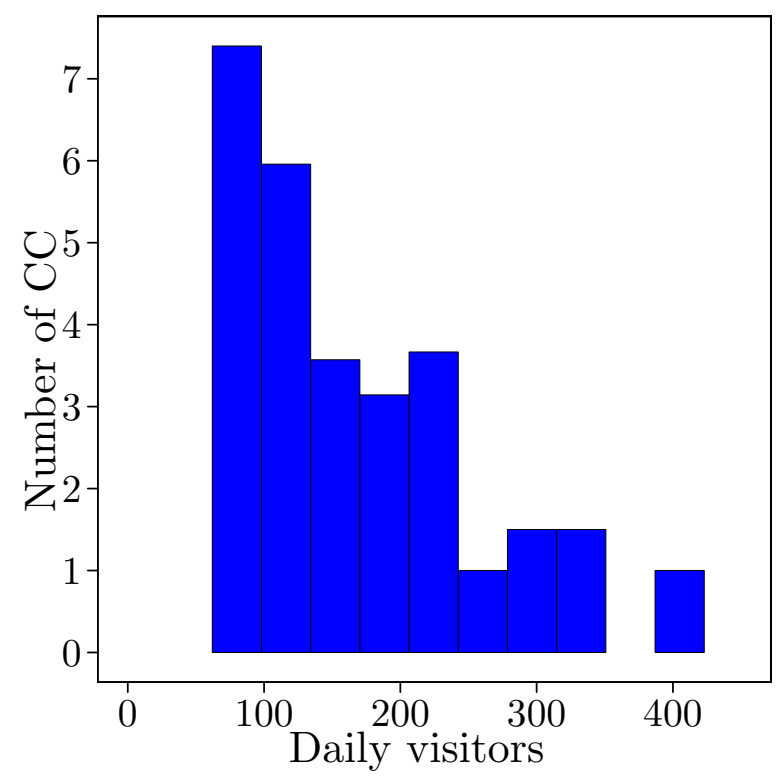

FIG. 2: Number of connected components (CCs) in the daily aggregated networks of the SG deployment as a function of the number of visitors.

particular the presence of either a single or two large connected components (CC) in the network. Days with smaller numbers of visitors can also give rise to aggregated networks made of a larger number of small isolated clusters. As shown in Fig. 2 depending on the number of visitors the number of CC can in fact vary substantially. For a large number of visitors, typically only one CC is observed. For a low number of visitors, on the other hand, many clusters are formed. Overall one also notices that the network diameter (highlighted in all the plots of Fig. 1 is considerably longer for SG than for HT09 aggregated networks, reflecting the different behavioral patterns in these settings.

The small-world nature — or lack thereof — of the aggregated networks can be investigated statistically by introducing a proper null model. To this end, we construct a randomized network using the rewiring procedure described in Ref. 39. The procedure consists in taking random pairs of links $(i, j)$ and $(l, m)$ involving 4 distinct nodes, and rewiring them as $(i, m)$ and $(j, l)$. This procedure preserves the degree of each node and the degree distribution $P(k)$, while destroying the degree correlations between neighboring nodes, as well as any other correlations linked to node properties. The procedure is carried out so that initially distinct CCs do not get merged. Since the rewiring procedure cannot be implemented for the rare CCs with less than four nodes, these small CCs are removed from the aggregated networks before rewiring. Figure 3 displays a single realization of the null model for the networks in the top row of Fig. 1. We notice that the rewired version of the aggregated HT09 network is very similar to the original version, whereas the null model for the aggregated network of the SG data on July $14^{\text {th }}$ is more "compact" than the original network and exhibits a much shorter diameter. Similar considerations hold for the other aggregated networks of the SG deployment.

More quantitatively, we measure the mean number of nodes one can reach from a randomly chosen node by making $l$ steps on the network, a quantity hereafter called $M(l)$. For a network consisting of a single connected component, the definition of $M(l)$ implies that

$$
M(1)=\langle k\rangle+1 \quad \text { and } \quad M(\infty)=N,
$$

where $\langle k\rangle$ is the average node degree, $N$ is the total number of nodes in the network and $M(\infty)$ the saturation value of $M$ on the network. The saturation value $M(\infty)$ is reached when $l$ is equal to the length of the network diameter, and may vary for different realizations of the random networks. For a network consisting of several CCs one has to take into account the probability $N_{i} / N$ that the chosen node belongs to a given CC, where $N_{i}$ is the number of nodes in the $i$-th CC. As a consequence, Eq. (1) generalizes to

$$
M(1)=\frac{1}{N} \sum_{i} N_{i}\left(\langle k\rangle_{i}+1\right) \text { and } M(\infty)=\frac{1}{N} \sum_{i} N_{i}^{2},
$$

where $\langle k\rangle_{i}$ is the average node degree on the $i$-th CC. This ensures that the quantity $M(l) / M(\infty)$, regardless on the number of $\mathrm{CC}$, assumes the same value when $l=1$, and saturates to unity for both the aggregated and rewired 
HT09: June $30^{\text {th }}$ (rewired)

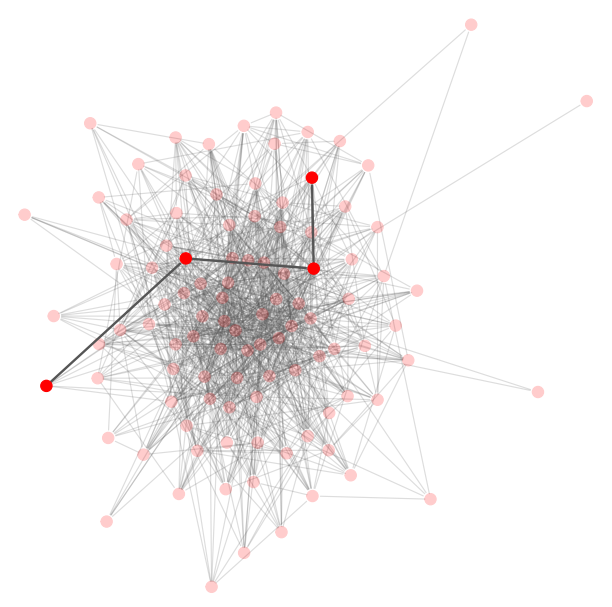

SG: July $14^{\text {th }}$ (rewired)

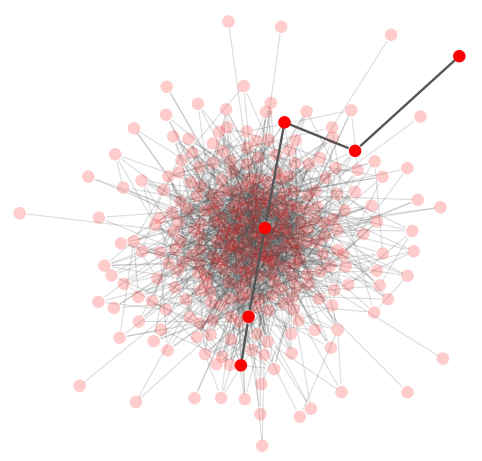

FIG. 3: Randomized versions of the daily aggregated networks in the top row of Fig. 1. Left: HT09 deployment, June $30^{\text {th }}$. Right: SG deployment, July $14^{\text {th }}$. The network diameters are highlighted as in Fig. 1] In the SG case, the randomized network is much more "compact" than the original one, with a much shorter diameter.

network. Figure 4 displays $M(l) / M(\infty)$ for the aggregated networks on the top row of Fig. 1, as well as its value averaged on 100 randomized networks (the average value of $M(l)$ converges rapidly already when calculated on a few tens of randomized networks). We notice the striking similarity between the results for the HT09 original and randomized networks, where about $90 \%$ of the individuals lie, in both cases, within two degrees of separation. In the SG case, conversely, the same $90 \%$ percentage is reached with six degrees of separation for the original network, but with only three degrees of separation on the corresponding randomized networks. The same calculation, performed on other aggregated SG networks, yields qualitatively similar results, always exposing a dramatic difference from the null model.

One of the standard observables used to characterize a network topology is the degree distribution $P(k)$, i.e., the probability that a randomly chosen node has $k$ neighbors. Figure 5 reports the degree distributions of the daily aggregated networks, averaged over the whole duration of the HT09 deployment (left) and SG deployment (right). For the SG case, we left out the few isolated nodes that contribute to the degree distribution for $k=0$ only. The $P(k)$ distributions are short-tailed in all cases: $P(k)$ decreases exponentially in the SG case, and even faster for HT09. We notice that the HT09 degree distribution exhibits a peak at $k$ around $15-20$, pointing to a characteristic number of contacts established during the conference. Moreover, the average degree in the HT09 case, $\langle k\rangle$, close to 20 , is more than twice as high as that for the SG networks, $\langle k\rangle$ which is close to 8. This represents another clear indication of the behavioral difference of conference participants versus museum visitors (the fact that the average degree is high for conference attendees can be regarded as a goal of the conference itself). Finally, we observe that a large fraction of the recorded contacts are sustained for a short time: for instance, removing all the contacts with a cumulated duration below one minute yields $\langle k\rangle$ about 7.5 for HT09 and $\langle k\rangle$ about 3.5 for SG.

\section{TEMPORAL FEATURES}

The availability of time-resolved data allows one to gain much more insight into the salient features of the social interactions taking place during the deployments than what could be possible by the only knowledge of "who has been in face-to-face proximity of whom".

We first investigated the presence duration distribution in both settings. For the conference case, the distribution is rather trivial, as it essentially counts the number of conference participants spending one, two or three days at the conference. The visit duration distribution for the museum, instead, can be fitted to a lognormal distribution (see Fig. 6), with geometric mean around 35 minutes. This shows that, unlike the case of the conference, here one can meaningfully introduce the concept of a characteristic visit duration that turns out to be well below the cutoff 

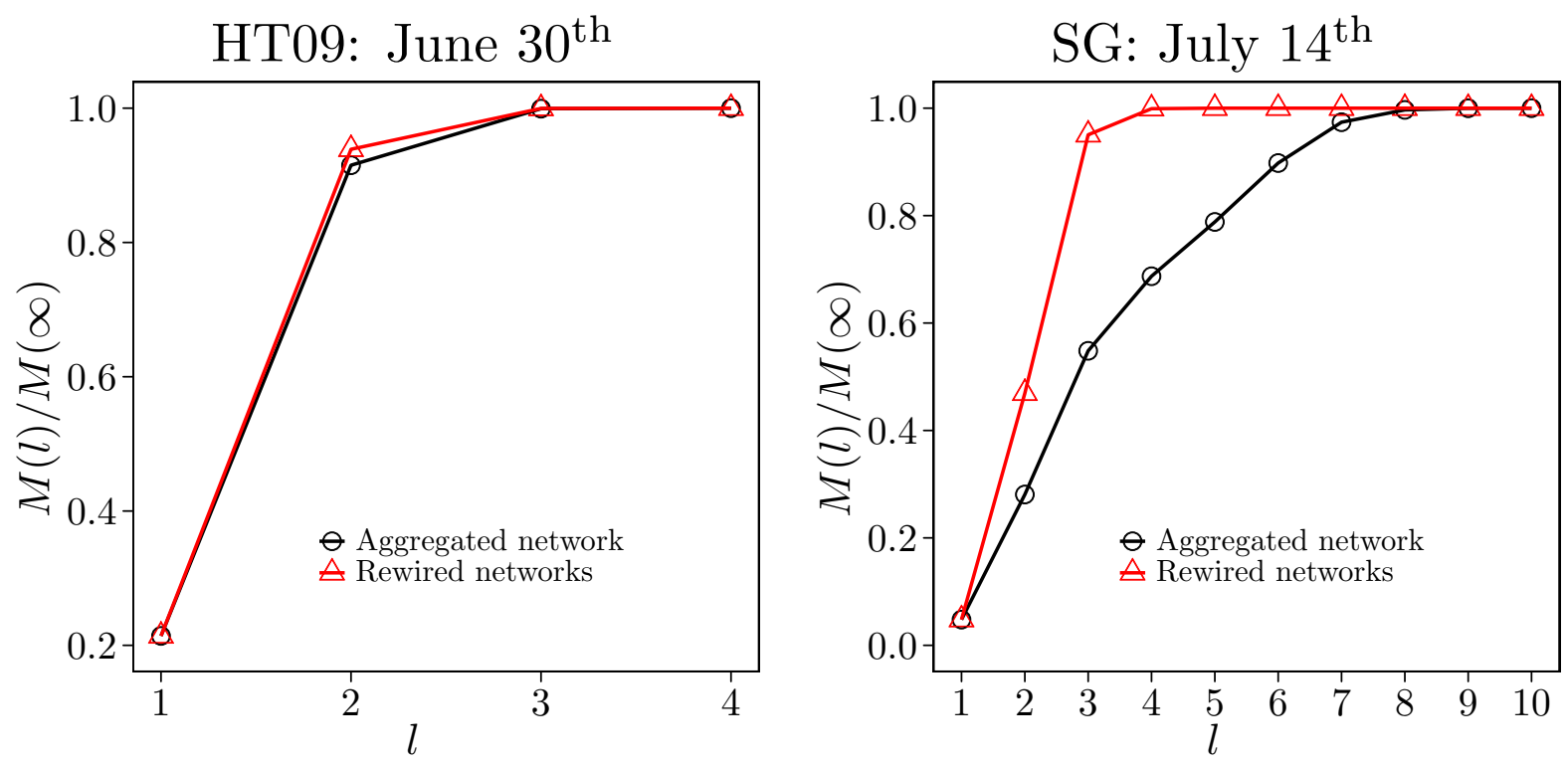

FIG. 4: Average number of nodes reachable from a randomly chosen node by making $l$ steps on the network, $M(l)$, divided by its saturation limit $M(\infty)$, for daily aggregated networks (circles) and their randomized versions (triangles). For the randomized case, data are averaged on 100 realizations. Left: network aggregated on June $30^{\text {th }}$ for the HT09 case. Right: SG deployment, July $14^{\text {th }}$. The solid lines are only guides for the eye.
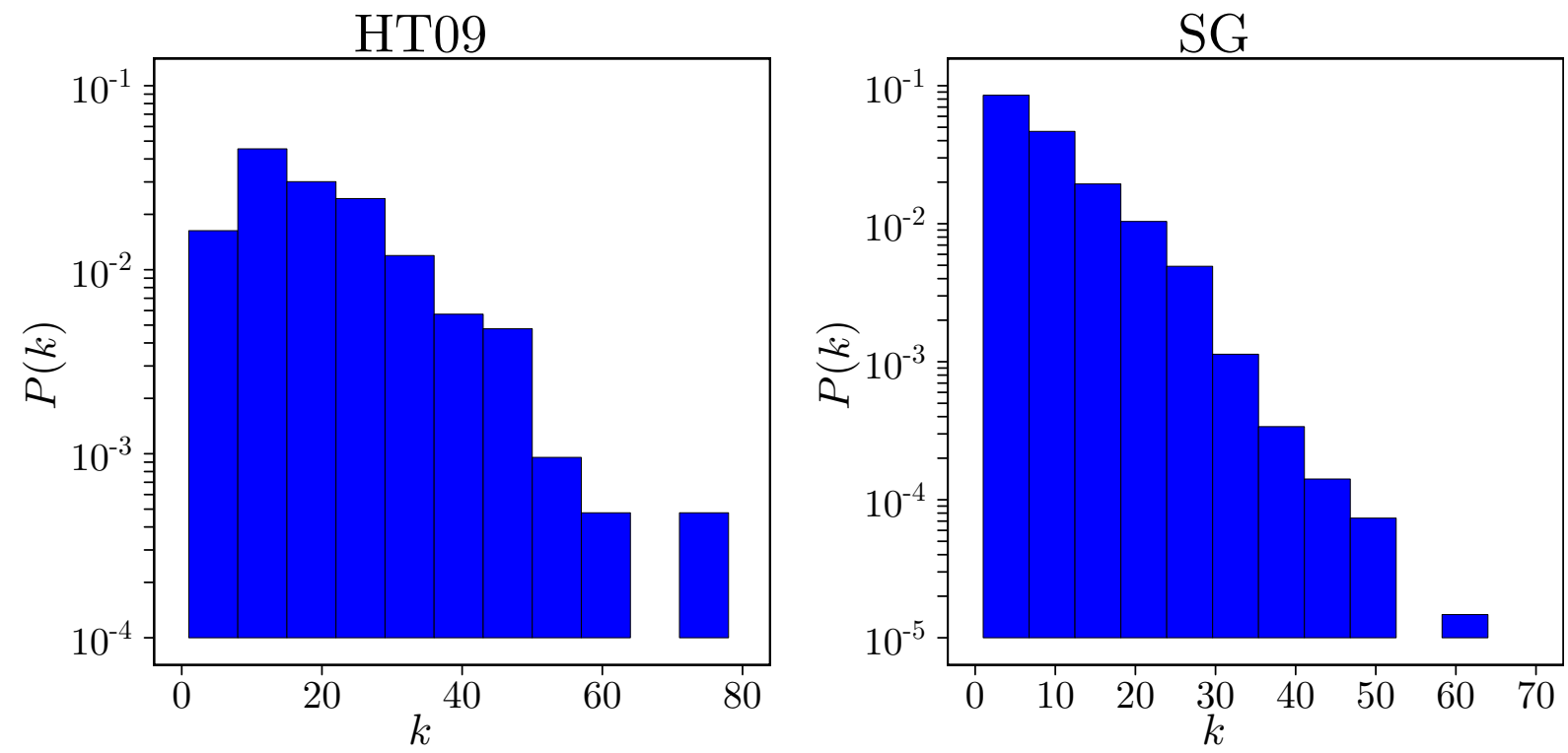

FIG. 5: Degree distributions $P(k)$ averaged over all daily aggregated networks, for the HT09 (left) and the SG (right) cases.

imposed by museum opening hours. The existence of a characteristic visit duration sheds light on the elongated aspect of the aggregated networks of visitor interactions (see Fig. 1). Indeed museum visitors are unlikely to interact directly with other visitors entering the venue more than one hour after them, thus preventing the aggregated network from exhibiting small-world properties. Figure 7 reports the SG aggregated networks for two different days, where the network diameter is highlighted and each node is colored according to the arrival time of the corresponding visitor. One notices that, as expected from the aforementioned properties of the visit duration distribution, there is limited interaction among visitors entering the museum at different times. Furthermore, the network diameter clearly defines a path connecting visitors that enter the venue at subsequent times, mirroring the longitudinal dimension of the 


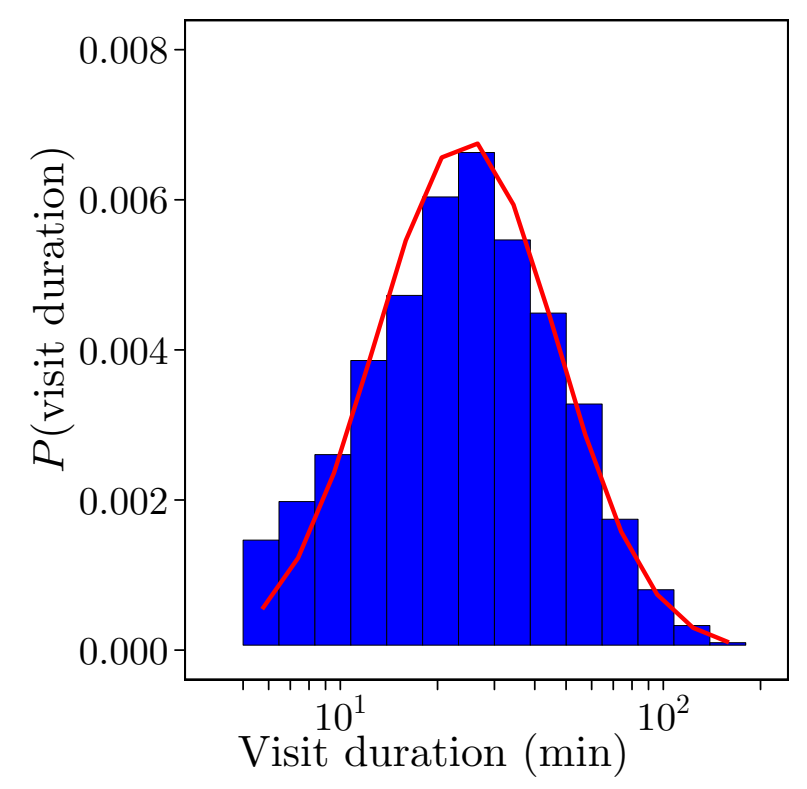

FIG. 6: Visit duration distribution at the SG museum (histogram) and fit to a lognormal distribution (red line).
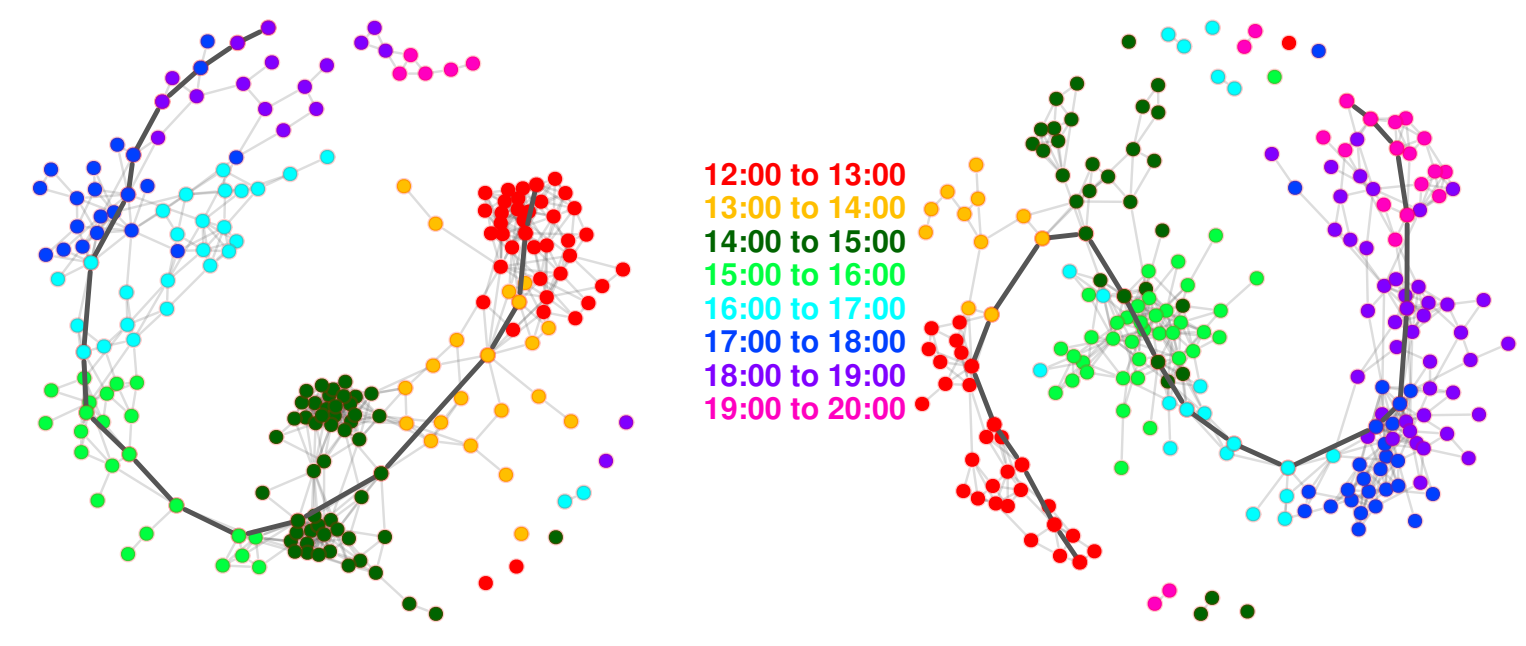

FIG. 7: (Color online) Aggregated networks for two different days of the SG museum deployment. Nodes are colored according to the corresponding visitor's entry time slot. The network diameter is highlighted in each case.

network. These findings show that aggregated network topology and longitudinal/temporal properties are deeply interwoven.

Let us now focus on the temporal properties of social interactions. At the most detailed level, each contact between two individuals is characterized by its duration. The corresponding distributions are shown in Fig. 8. As noted before, in both the HT09 and SG cases most of the recorded interactions amount to shortly-sustained contacts lasting less than one minute. However, both distributions show broad tails - they decay only slightly faster than a power law. This behavior does not come as a surprise, as it has been observed in social sciences in a variety of context ranging from human mobility to email or mobile phone calls networks [16, 22, 28, 40. More interestingly, the distributions are 


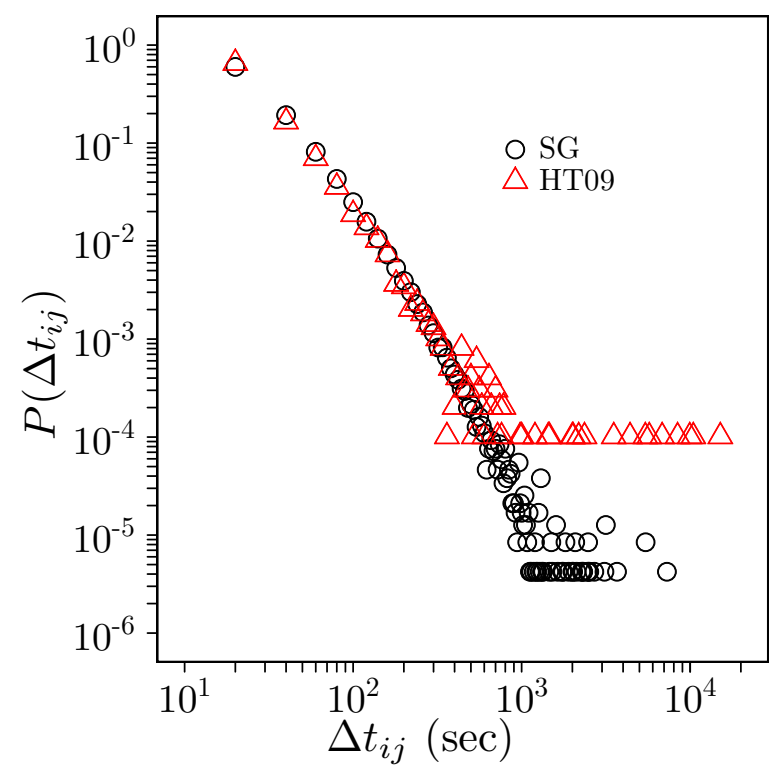

FIG. 8: Distributions of the contact durations for the HT09 (triangles) and SG (circles) deployments, averaged over all days. Despite the differences in the measurement contexts, the distributions are superimposed.

very close (except in the noisy tail, due to the different number of contributing events), showing that the statistics of contact durations are robust across two very different settings. This robustness has been observed in Ref. 28] across different scientific conferences, but the museum setting corresponds to a situation in which a flux of individuals follows a predefined path, and this strong similarity between distributions was therefore not expected a priori. At a coarser level, aggregated networks are characterized by weights on the links, that quantify for how long two individuals have been in face-to-face proximity during the aggregation interval. Figure 9 displays the distributions of these weights $w$. These distributions are very broad 28] : while most links correspond to very short contacts, some correspond to very long cumulated durations, and all time scales are represented, that is, no characteristic interaction timescale (except for obvious cutoffs) can be determined. We note that at this coarser level of analysis the distributions are again very similar.

For each individual, the cumulated time of interaction with other individuals is moreover given by the strength $s$ of the corresponding node 12, i.e., by the sum of the weights of all links inciding on it. The strength distributions $P(s)$ are displayed in Fig. 10 for the aggregated networks of the HT09 conference (left) and of the SG museum case (right). Unlike $k$, the node strength $s$ spans several orders of magnitude, ranging from a few tens of seconds to well above one hour. The node strength $s$ can be correlated with the node degree $k$ by computing the average strength $\langle s(k)\rangle$ of nodes of degree $k$ [12. While a completely random assignment of weights yields a linear dependency with $\langle s(k)\rangle$ proportional to $\langle w\rangle k$, where $\langle w\rangle$ is the average link weight, super-linear or sub-linear behaviors have been observed in various contexts [12, 28, 41]. A super-linear dependence such as the one observed in some conference settings 28 hints at the presence of super-spreader nodes that play a prominent role in processes such as information diffusion [42, 43]. On the other hand, the sub-linear dependence observed for large-scale phone call networks [4] corresponds to the fact that more active individuals spend on average less time in each call. Figure 11 displays the ratio $\langle s(k)\rangle /(\langle w\rangle k)$ for the SG and HT09 daily aggregated networks. Two different trends appear despite the large fluctuations: a slightly increasing trend in the conference setting, and a clearly decreasing one in the museum setting. In particular, the behavior of $\langle s(k)\rangle /(\langle w\rangle k)$ for the HT09 case (left plot in Figure 11) can be fitted linearly yielding a linear coefficient $\Delta=0.01$ (p-value $=0.007)$. By reshuffling 4000 times the weights of the network links and performing the same linear fit for each reshuffling, we obtain a distribution of linear coefficients $\Delta$. Such distribution, whose mean is zero, is shown in the inset of the left plot in Figure 11 together with the value of $\Delta$ from the HT09 daily aggregated networks (vertical line). The observed value of $\Delta$ at the HT09 is an outlier of the distribution ( $96^{\text {th }}$ percentile), thus showing that the observed behavior of $\langle s(k)\rangle /(\langle w\rangle k)$ can hardly arise by a random assignment of link weights. On the other hand, the observed behavior of $\langle s(k)\rangle /(\langle w\rangle k)$ at the $\mathrm{SG}$ can be fitted to a power law with a negative exponent i.e. it decreases linearly on a double logarithmic scale such as the one shown in the inset of the right plot in Figure 11 . These results indicate that individuals who encountered the same number of distinct persons can have different spreading potentials, depending on the setting. It also gives a warning about characterizing spreading by only measuring the 


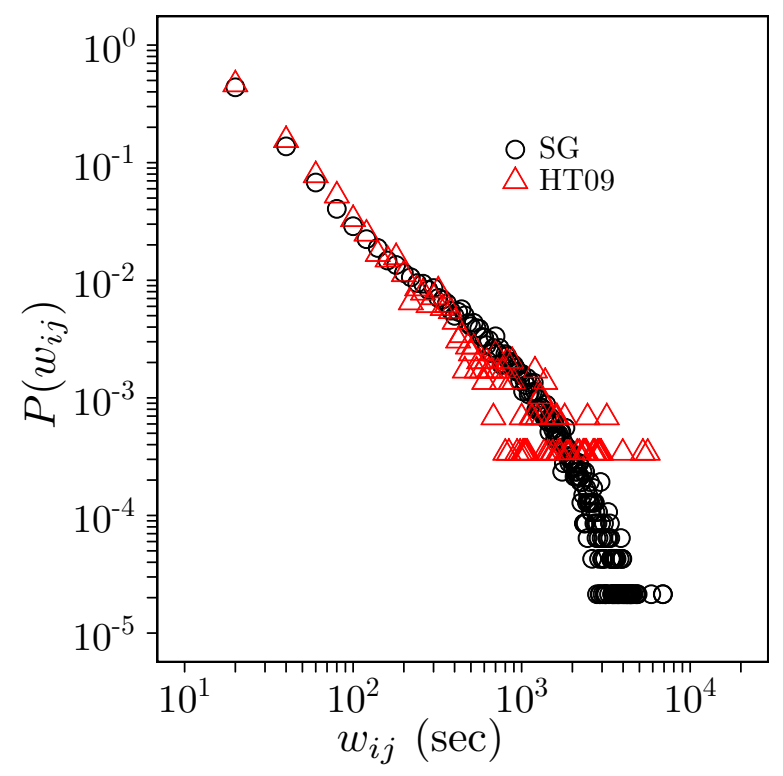

FIG. 9: Weight distributions for the daily aggregated networks of one HT09 conference day (triangles) and for the SG aggregated networks (circles), averaged over all daily aggregated networks. The weight of a link represents the total time spent in face-toface proximity by the two linked individuals during the aggregation interval (here one day).
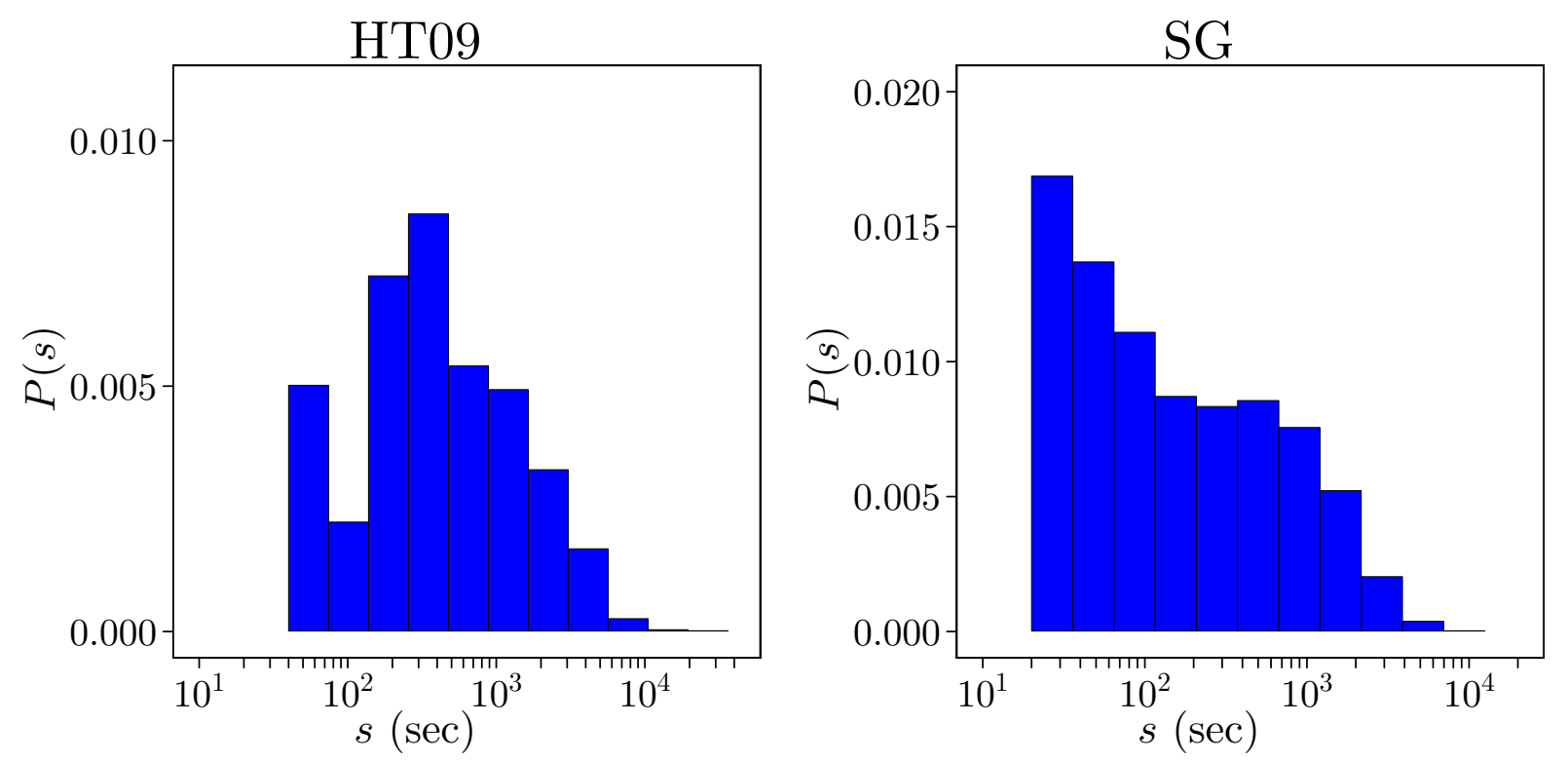

FIG. 10: Strength distributions $P(s)$ in the HT09 (left) and SG (right) aggregated networks (data for all daily networks). The strength of a node quantifies the cumulated time of interaction of the corresponding individual with other individuals.

number of encounters, which can yield a rather misleading view.

\section{PERCOLATION ANALYSIS}

The issue of network vulnerability to successive node removal has attracted a lot of interest in recent years starting from the pioneering works of Refs. 44, 45, that have shown how complex networks typically retain their integrity when nodes are removed randomly, while they are very fragile with respect to targeted removal of the most connected 

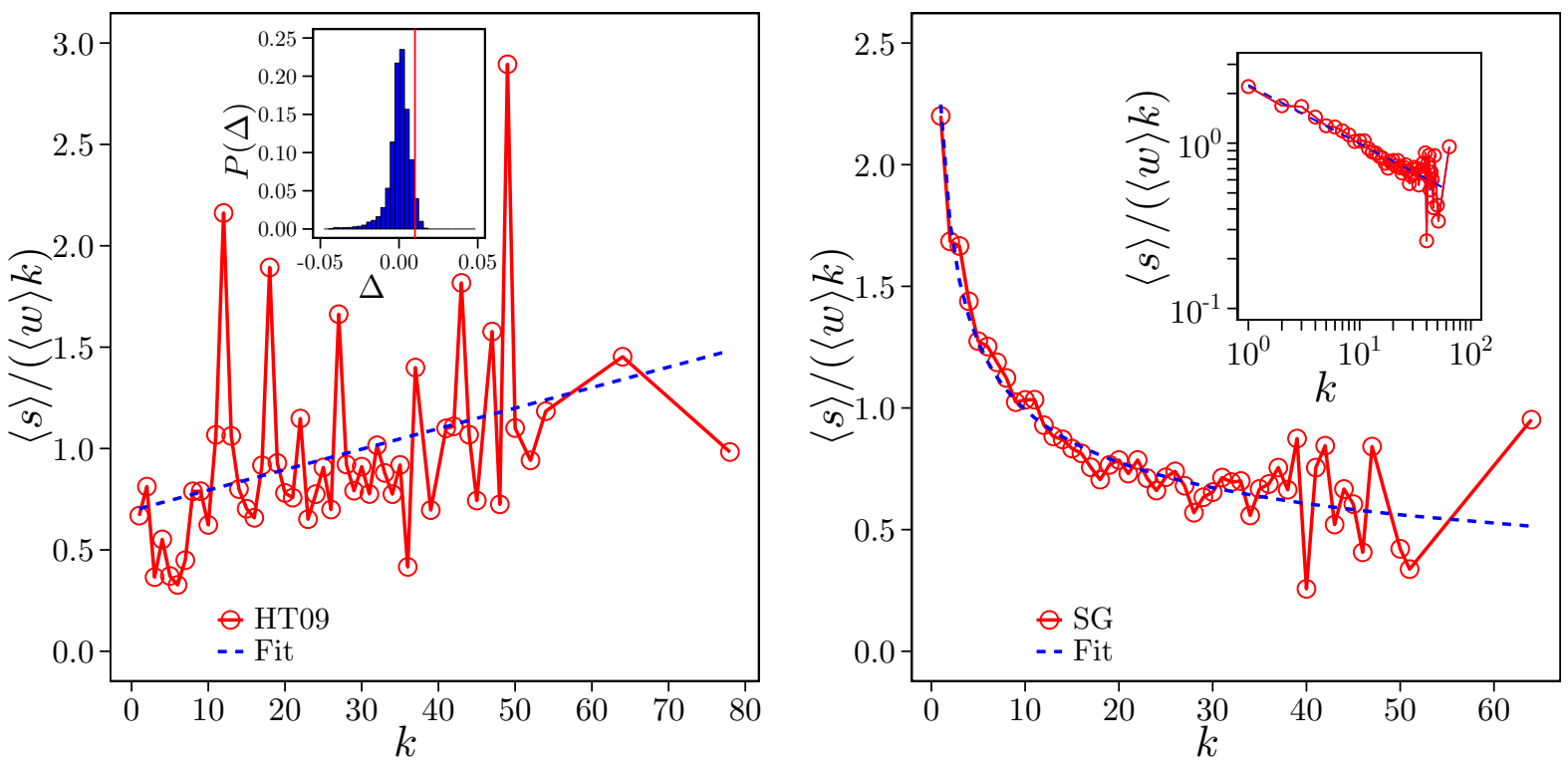

FIG. 11: (Color online) Correlation between node's strength and degree, as measured by the average strength $\langle s(k)\rangle$ of nodes of degree $k$. The figures show $\langle s(k)\rangle /(\langle w\rangle k)$ (circles), for the HT09 (left) and SG (right) deployments (the solid line is only a guide for the eye). The dashed lines stand for a linear fit and a power law fit to the data for the HT09 and SG deployments, respectively. Distinct increasing and decreasing trends are respectively observed. The inset for the HT09 deployment shows a distribution of linear coefficients $\Delta$ calculated for 4000 reshufflings of the network weights and the fitted value from the data collected at HT09 (vertical line). The inset for the SG deployment shows $\langle s(k)\rangle /(\langle w\rangle k)$ on a doubly logarithmic scale (circles) together with the power law fit to the data (dashed line).

nodes. While the concepts of node failures and targeted attacks are pertinent for infrastructure networks, successive removals of nodes or links is more generally a way to study network structures [46 49. For instance, detecting efficient strategies for dismantling the network sheds light on the network community structure, as it amounts to finding the links that act as bridges between different communities [47, 48. Moreover, in the context of information or disease spreading, the size of the largest connected component gives an upper bound on the number of nodes affected by the spreading. Identifying ways to reduce this size, by removing particular links, in order to break and disconnect the network as much as possible, is analogous in terms of disease spreading to finding efficient intervention and containment strategies.

In order to test different link removal strategies, we consider different definitions of weight for a link connecting nodes $i$ and $j$ in the aggregated contact network:

- The simplest definition of link weight is given by the cumulated contact duration $w_{i j}$ between $i$ and $j$. In the following, we will refer to this weight as "contact weight".

- The topological overlap $O_{i j}$, introduced in Ref. [50, is defined as

$$
O_{i j}=\frac{n_{i j}}{\left(k_{i}-1\right)+\left(k_{j}-1\right)-n_{i j}} \in[0,1],
$$

where $k_{i(j)}$ is the degree of node $i(j)$ and $n_{i j}$ measures the number of neighbors shared by nodes $i$ and $j$. This measure is reminiscent of the edge clustering coefficient [48, and evaluates the ratio of the number of triangles leaning upon the $i j$ edge with the maximum possible number of such triangles given that $i$ and $j$ have degrees $k_{i}$ and $k_{j}$, respectively. Edges between different communities are expected to have a low number of common neighbors, hence a low value of $O_{i j}$.

- Finally, the structural similarity of two nodes is defined as the cosine similarity

$$
\operatorname{sim}_{i j}=\frac{\sum_{l \in \mathcal{V}} w_{i l} w_{j l}}{\sqrt{\sum_{l} w_{i l}^{2} \sum_{l} w_{j l}^{2}}} \in[0,1],
$$

where $\mathcal{V}$ is the set of neighbors shared by nodes $i$ and $j$, and the sums at the denominator are computed over all the neighbors of $i$ and $j$. Cosine similarity, which is one of the simplest similarity measures used in the field of information 
retrieval [52 54, takes into account not only the number of shared neighbors of $i$ and $j$, but also the similarity of the corresponding edge strengths, i.e. the similarity of individuals in terms of the time they spent with their neighbors. Once again, edges connecting different communities are expected to have a low value of $\operatorname{sim}_{i j}$.

Based on these three weight definitions, we consider four different strategies for link removal, namely: removing the links in increasing/decreasing order of contact weight, in increasing order of topological overlap, and in increasing order of cosine similarity. The former two strategies are the simplest one can devise, as they do not consider the neighborhoods' topology. The latter two strategies were implemented in an incremental fashion, by recomputing the lists of links ranked in order of increasing overlap or cosine similarity whenever a link was removed, and then removing the links in the updated list order [57]. An issue also arises from the fact that all the generalized weights mentioned above produce a certain amount of link degeneracy (in particular when using the contact weight): for instance, many links may have the same (small) value $w_{i j}$, or exactly 0 overlap or similarity. Each link removal procedure carries therefore a certain ambiguity, and the results may depend on which links, among those with the same contact weight/overlap/similarity, are removed first.

The impact of link removal on network fragmentation can be measured by monitoring the variations of the size of the largest CC, hereafter called $N_{1}$, as a function of link removal. If the network is initially divided into two CCs, labeled $C_{1}^{0}$ and $C_{2}^{0}$, of similar initial sizes $N_{1}^{0} \geq N_{2}^{0}$, we call $N_{1}$ the size of the largest CC surviving in the network (which does not need to be a subnetwork of $C_{1}^{0}$ ). We used the apex " 0 " to denote quantities expressed for the original network, before any link removal. In order to alleviate the problems arising from link degeneracy, we averaged $N_{1}$ on 100 different link orderings (i.e. we reshuffled the list of links of equal generalized weight before removing them).

An example of a single realization of the removal strategies for the SG aggregated network of July $14^{\text {th }}$ is shown in Fig. 12. We observe that a removal of $60 \%$ of the network links has a far deeper impact on the network when the removal is based on the topological overlap (the size of the largest CC is $N_{1}=30$ ) or cosine similarity $\left(N_{1}=155\right)$ rather than on decreasing (increasing) contact weight $\left(N_{1}=204(205)\right)$. More quantitatively, Fig. 13 shows that removing links according to their topological overlap is the most efficient strategy. This is in agreement with previous results 46 .50] that have shown that topological criteria detect efficiently the links that act as bridges between communities. Due to their high degeneracy, removing first the links with small contact weights approximates a random removal strategy that is far from optimal. Despite this limitation, removing the links with small contact weights can outperform the removal of links with high contact weight since the latter are usually found within dense communities, while links between communities have typically small contact weights.

The strategy based on link topological overlap proves slightly more effective than the strategy based on link similarity: the information on the link contact weights incorporated in the definition of $\operatorname{sim}_{i j}$ (Eq. 4 ) does not enhance the decrease of $N_{1}$. This can be explained through the following argument: topological overlap link ranking usually leads to a higher degeneracy with respect to similarity-based link ranking. As a consequence, for a network with similar values of $N_{1}^{0}$ and $N_{2}^{0}$, a strategy based on topological overlap is more likely to dismantle in parallel both $C_{1}^{0}$ and $C_{2}^{0}$ than a similarity-based strategy, as it has no bias towards a specific component. The opposite strategy of a complete dismantling of $C_{1}^{0}$ that leaves $C_{2}^{0}$ intact would result in $N_{1}=N_{2}^{0}$ even after the complete disintegration of $C_{1}^{0}$. This effect is illustrated in Fig. 13 for the SG aggregated networks of May $19^{\text {th }}-20^{\text {th }}$, which are indeed composed of two large CCs (see Fig. 11).

Interestingly, and as expected from the previous comparisons, rather different results are obtained for the HT09 and SG aggregated networks. The conference network is more resilient to all strategies, and significant levels of disaggregation are reached only by removing large fractions $(\geq 40-60 \%)$ of the links, sorted by their topological overlap. For the SG aggregated networks, on the other hand, targeting links with small topological overlap or cosine similarity is a quite effective strategy, which can be intuitively related to the modular structure visible in Fig. 1 .

\section{DYNAMICAL SPREADING OVER THE NETWORK}

Aggregated networks often represent the most detailed information that is available on social interactions. In the present case, they would correspond to information obtained through ideal surveys in which respondents remember every single person they encountered and the overall duration of the contacts they had with that person. While such a static representation is already informative, it lacks information about the time ordering of events, and it is unable to encode causality. The data from our measurements do not suffer from this limitation, as they comprise temporal information about every single contact. Therefore, these data can be used to investigate the unfolding of dynamical processes. They also allow to study the role of causality in diffusion processes, such as the spreading of an infectious agent or of a piece of information on the encounter networks of individuals. In the following we will mainly use an epidemiological terminology, but we may equally imagine that the RFID devices are able to exchange some information whenever a contact is established. Individuals will be divided into two categories, susceptible individuals (S) or infected ones (I): susceptible individuals have not caught the "disease" (or have not received the information), 

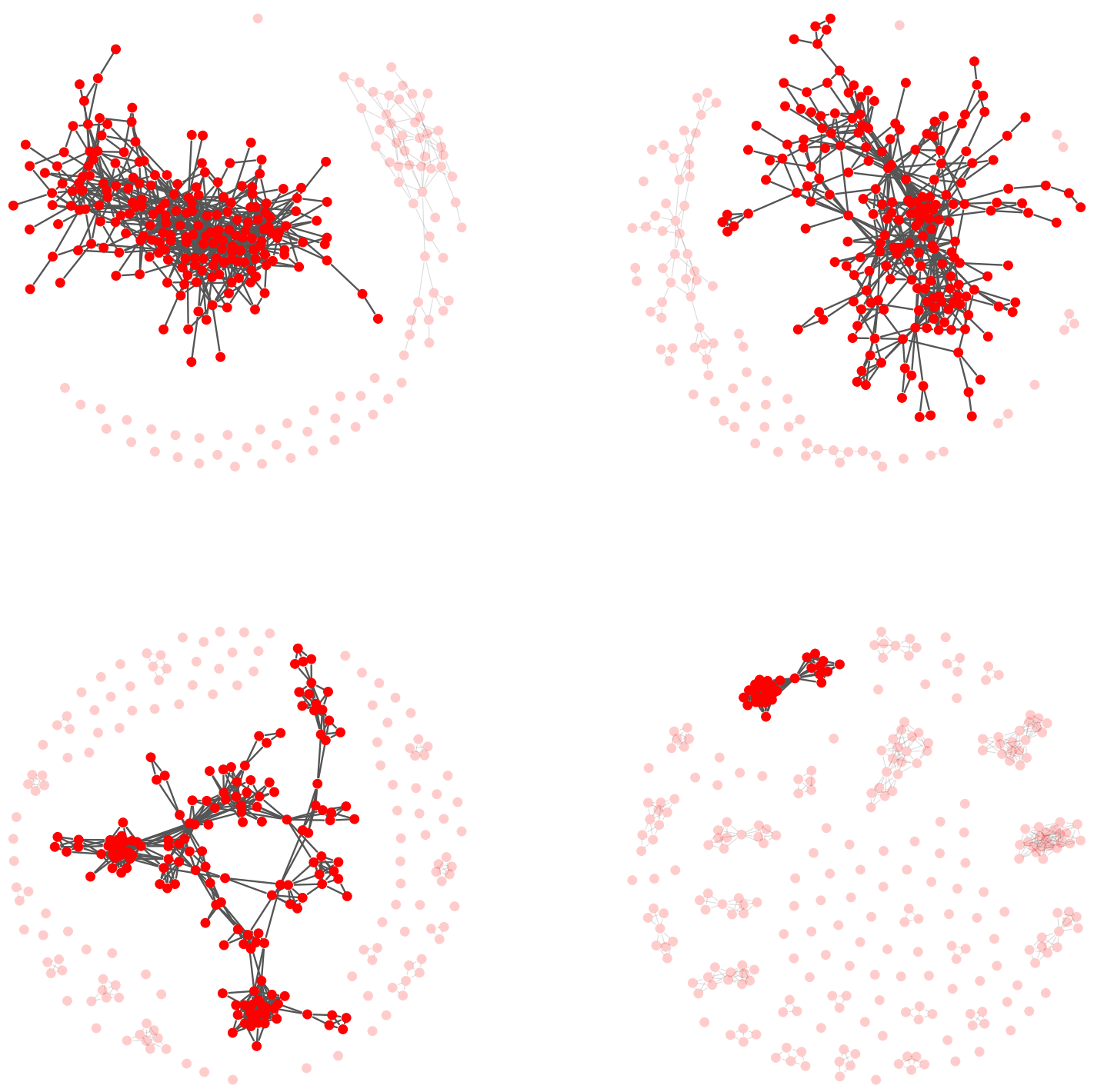

FIG. 12: Effect of four different ways of removing $60 \%$ of the links on the SG museum daily aggregated network of July $14^{\text {th }}$. Clockwise from top: links removed in decreasing contact weight order, increasing contact weight order, increasing topological overlap order and increasing cosine similarity order. The largest CC is highlighted in each case.

while infected ones carry the disease (or have received the information) and can propagate it to other individuals.

In order to focus on the structure of the dynamical network itself, we consider in the following a deterministic snowball SI model 43]: every contact between a susceptible individual and an infected one, no matter how short, results in a transmission event in which the susceptible becomes infected, according to $S+I \rightarrow 2 I$. In this model, individuals, once infected, do not recover. Such a deterministic model allows to isolate the role played in the spreading process by the structure of the dynamical network (e.g. its causality). Its role would otherwise be entangled with the stochasticity of the transmission process and the corresponding interplay of timescales. Of course, any realistic epidemiological model should include a stochastic description of the infection process, since the transmission from an infected individual to a susceptible one is a random event that depends on their cumulative interaction time. The resulting dynamics would depend on the interplay between contact and propagation times. We leave the study of this interesting type of interplay to future investigations.

In our numerical experiments, for each day we select a single "seed", i.e., an individual who first introduces the 

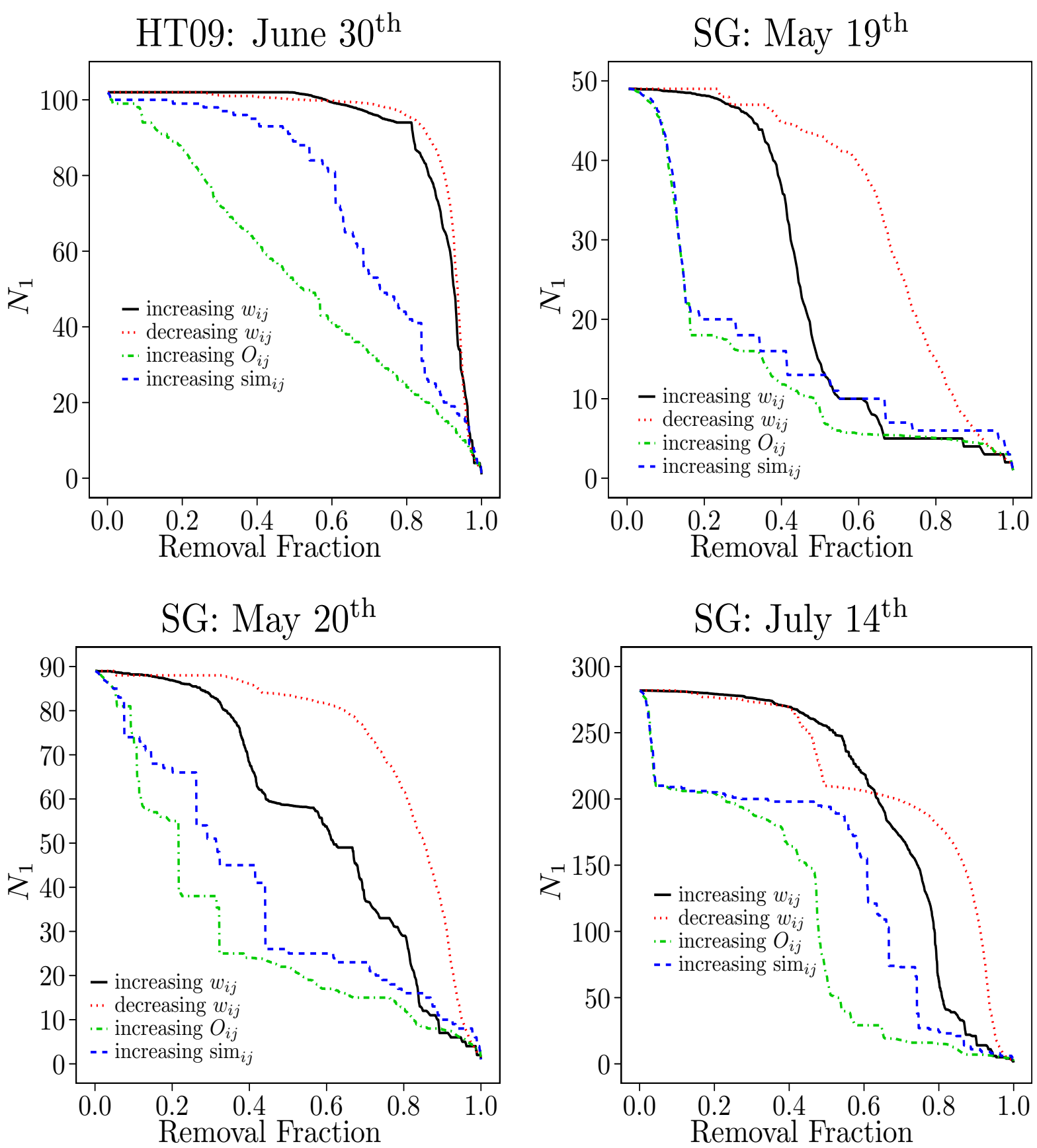

FIG. 13: (Color online) Size $N_{1}$ of the largest CC as a function of the fraction of removed links, for several removal strategies, and for different daily aggregated networks in the HT09 and SG deployments. For all networks, removing links in increasing topological overlap order and increasing cosine similarity order have the most disruptive effects. The HT09 aggregated network is in all cases more resilient than the SG aggregated networks.

infection into the network. All the other individuals are susceptible and the infection spreads deterministically as described above. By varying the choice of the seed over individuals, we obtain the distribution of the number of infected individuals at the end of each day. The transmission events can be used to define the network along which the infection spreads (i.e., the network whose edges are given by $S \leftrightarrow I$ contacts), hereafter called the transmission network.

Due to causality, the infection can only reach individuals present at the venue after the entry of the seed. As a consequence, in the following we will use the term partially aggregated network to indicate the network aggregated 

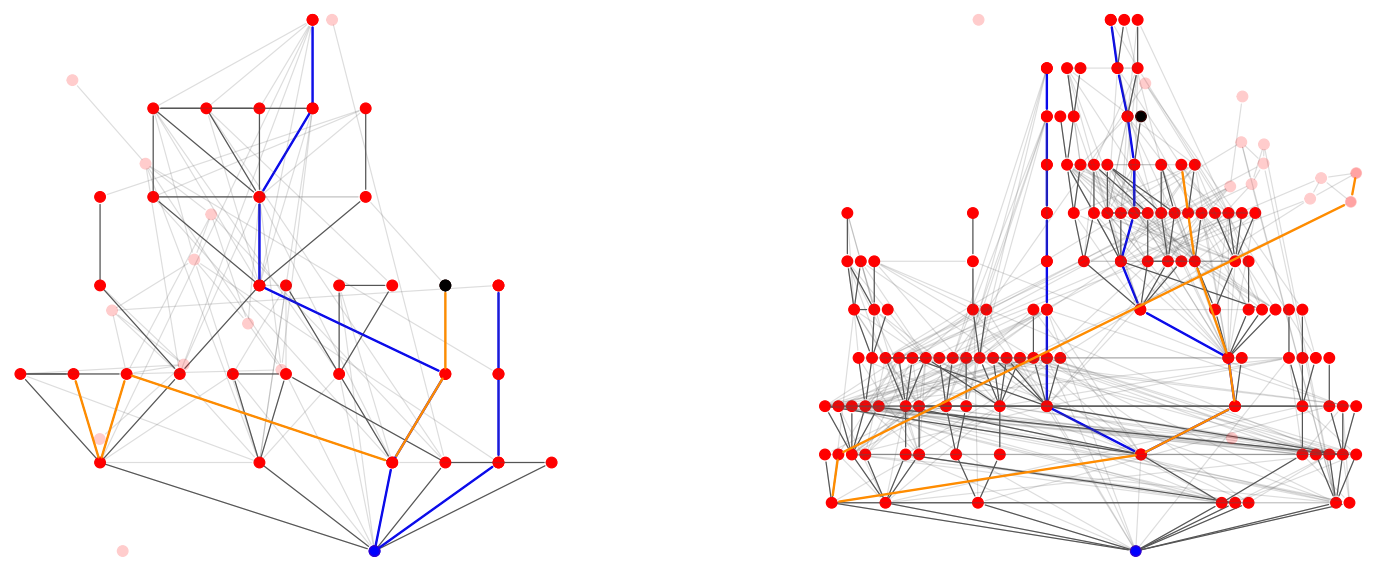

FIG. 14: (Color online) Partially aggregated networks on July $14^{\text {th }}$ at the SG museum, for two different choices of the seed (blue node at the bottom). Transparent nodes and light gray edges represent individuals not infected and contacts not spreading the infection, respectively. Red nodes and dark gray links represent infected individuals and contacts spreading the infection, respectively. The diameter of the transmission network and of the partially aggregated networks are shown respectively with blue and orange links. The black node represents the last infected individual.

from the time the seed enters the museum/conference to the end of the day. We note that the partially aggregated network defined in this way can be radically different from (much smaller than) the network aggregated along the whole day.

Figure 14 shows two partially aggregated networks for July $14^{\text {th }}$ at the SG museum, for two different choices of the seed (blue node), and the corresponding transmission networks. The transmission network is of course a subnetwork of the partially aggregated network: not all individuals entering the premises after the seed can be reached from the seed by a causal path, and not all links are used for transmission events. In order to emphasize the branching nature of infection spreading, we represent the transmission network with successively infected nodes arranged from the bottom to the top of the figure. We notice that the diameter of both the transmission and the partially aggregated network may not include the seed and/or the last infected individual.

The presence of a few triangles in the transmission network is due to the finite time resolution of the measurements. Let us consider, for instance, the case of an infected visitor $A$ who infects $B$, followed by a simultaneous contact of $A$ and $B$ with the susceptible $C$. In this case it is impossible to attribute the infection of $C$ to either $A$ or $B$, and both the $C \leftrightarrow A$ and the $C \leftrightarrow B$ links are highlighted in the transmission network as admissible transmission events. As a consequence, we slightly overestimate the number of links in the transmission network of Fig. 14. In the case of Fig. 14, the number of links is between 1 and $8 \%$ larger than for a tree with the same number of nodes. At finer time resolutions, some of the diffusion paths of Fig. 14 would actually be forbidden by causality.

A general feature exemplified by Fig. 14 is that the diameter of the transmission network (blue path) is longer than the diameter of the partially aggregated network (orange path), a first signature of the fact that the fastest paths between two individuals, which are the ones followed by the spreading process, do not coincide with the shortest path over the partially aggregated network [51].

The difference between the fastest and the shortest paths for a spreading process can be quantitatively investigated. Figure 15 reports the distribution of the network distances $n_{d}$ between the seed and every other infected individual along both the transmission networks and the aggregated networks. When calculated on the partially aggregated network, $n_{d}$ measures the length of the shortest seed-to-infected-individual path, whereas it yields the length of the fastest seed-to-infected-individual path when calculated on the transmission network. We observe that the length distribution of fastest paths, i.e., the $P\left(n_{d}\right)$ distribution for the transmission network, always turns out to be broader and shifted toward higher values of $n_{d}$ than the corresponding shortest path distribution, i.e., $P\left(n_{d}\right)$ for the partially aggregated network. The difference is particularly noticeable in the case of May $20^{\text {th }}$ and July $14^{\text {th }}$ for the SG deployment, and June $30^{\text {th }}$ for the HT09 conference, where the longest paths on the transmission network are about twice as long as the longest paths along the partially aggregated network. 

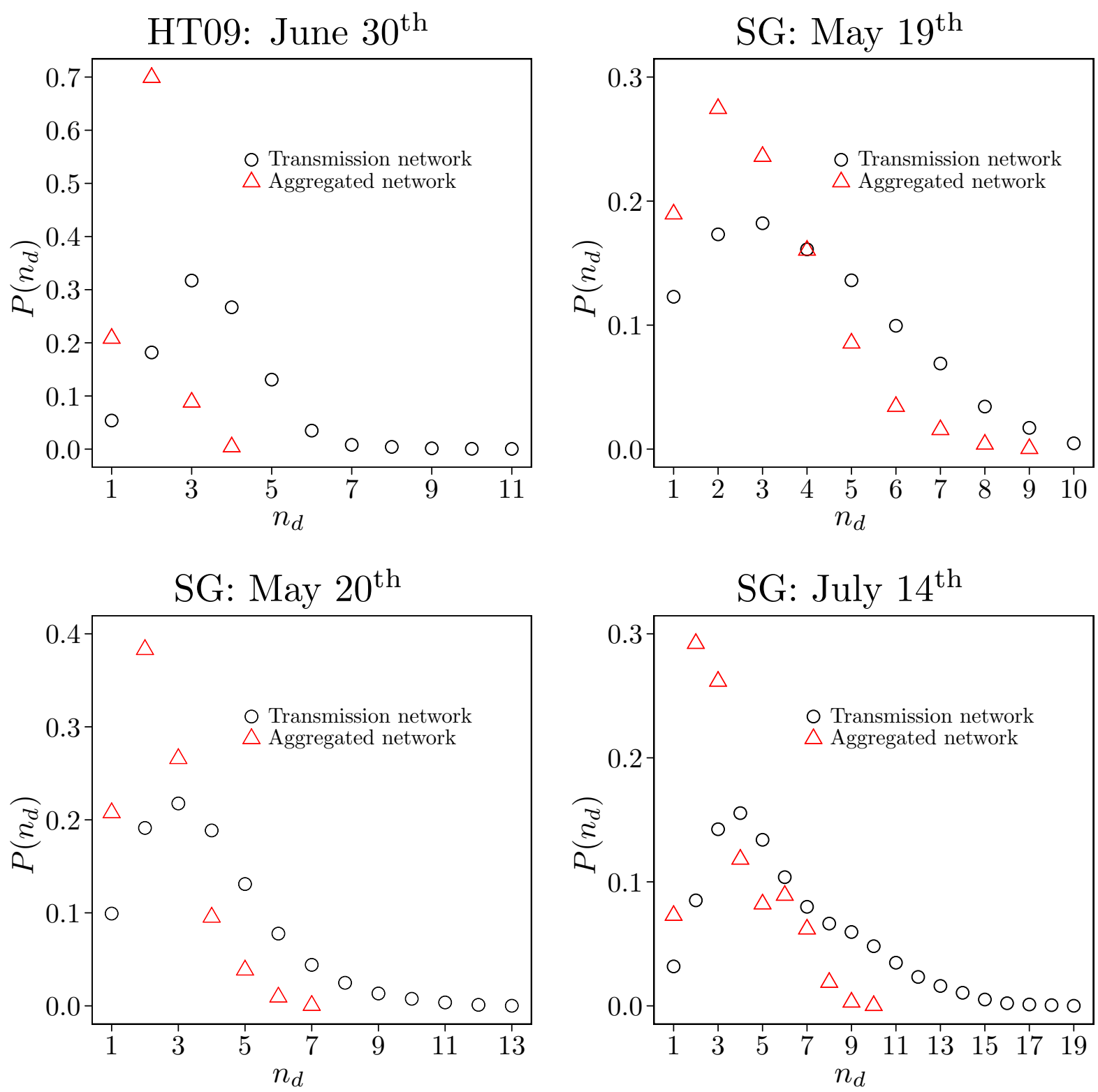

FIG. 15: (Color online) Distribution of the path lengths $n_{d}$ from the seed to all the infected individuals calculated over the transmission network (circles) and the partially aggregated networks (triangles). The distributions are computed, for each day, by varying the choice of the seed over all individuals.

These results clearly underline that in order to understand realistic dynamical processes on contact networks, information about the time ordering of the contact events turns out to be essential: the information carried by the aggregated network may lead to erroneous conclusions on the spreading paths.

It is also possible to study the length of the path connecting the first (seed) to the last infected individual along the transmission network. We measure the fastest seed-to-last-infected-individual path (a quantity hereafter called "transmission $n_{d}$ ") as a function of the duration of the spreading process, defined as the time between the entry of the seed and the last transmission event. As shown by Fig. 16. a clear correlation is observed between the transmission $n_{d}$ and the duration of the spreading process for the SG case (Pearson coefficients 0.76 for May $20^{\text {th }}$ and May $19^{\text {th }}$, and 0.9 for July $\left.14^{\text {th }}\right)$. No significant correlation is instead observed for the HT09 conference. This highlights the importance of the longitudinal dimension in the SG data, and gives a first indication of the strong differences in the spreading patterns, that we further explore in the following.

Let us now consider some other quantitative properties of the spreading process, in particular the number of 

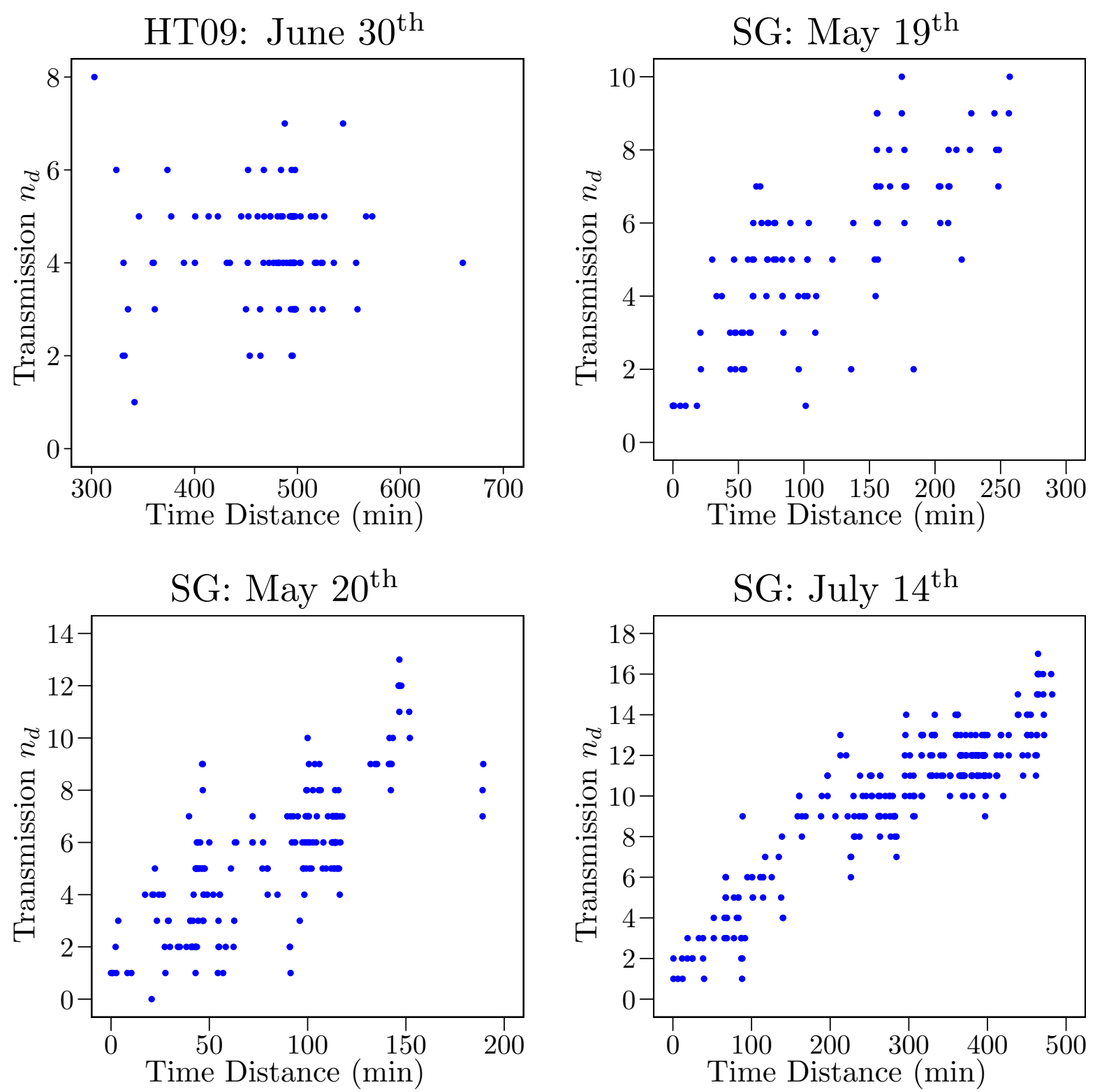

FIG. 16: Scatterplot of the seed-to-last-infected-individual distance (transmission $n_{d}$ ) along the transmission network, versus the total duration of the epidemics (time interval from the entry of the seed to the last infection event).

individuals reached by the infection/information at the end of one day. In the SG case Fig. 17 shows the distributions for each day, as boxplots, displaying the median together with the $5^{\text {th }}, 25^{t h}, 75^{\text {th }}$ and $95^{\text {th }}$ percentiles. Days are arranged horizontally from left to right, in increasing number of visitors. A high degree of heterogeneity is visible. The blue line corresponds to the number of daily visitors, that is the maximum number of individuals who can potentially be infected. We observe that the number of infected individuals is usually well below this limit. The number of reached individuals also depends on the number of CC in the aggregated network, as the spreading process cannot propagate from one $\mathrm{CC}$ to another. In fact, the limit for which all visitors are infected can be reached only if the aggregated network is globally connected, that occurs only when the global number of visitors is large enough. These results hint at the high intrinsic variability of the final outcome of an epidemic-like process in a situation where individuals stream through a building. A totally different picture emerges for the HT09 conference, where the infection is almost always able to reach all the participants.

As mentioned previously, the spreading process cannot reach individuals who have left the venue before the seed enters, or the individuals who belong to a $\mathrm{CC}$ different from that of the seed. Therefore, we consider the ratio of the 


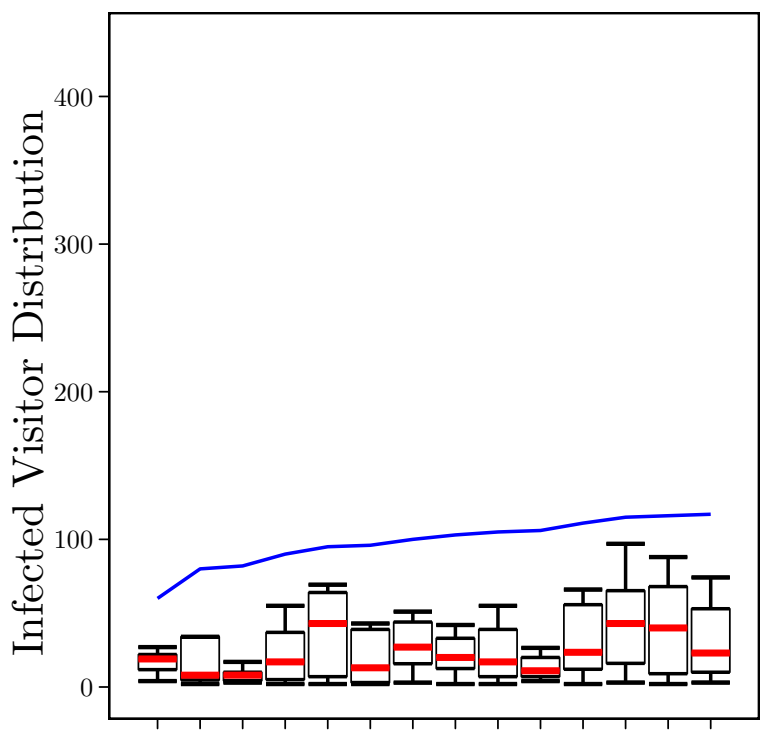

$60 \begin{array}{lllllll}60 & 82 & 90 & 95 & 96 & 100103105106111115116117\end{array}$

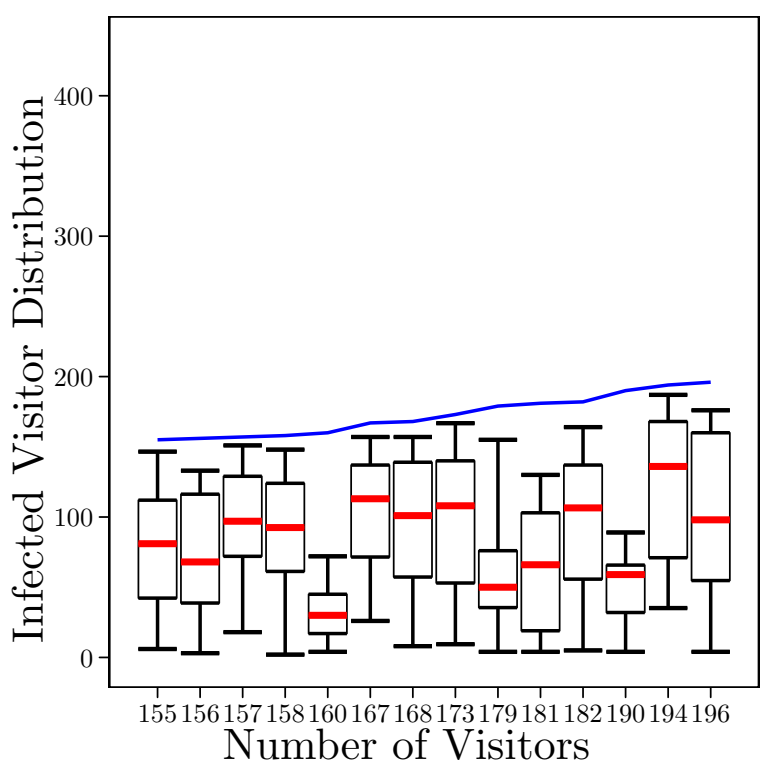

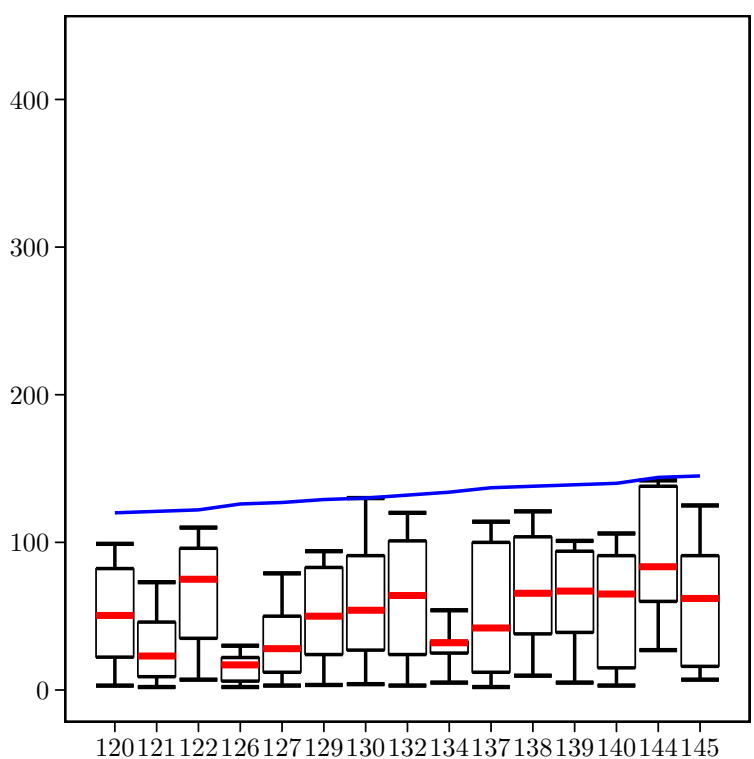

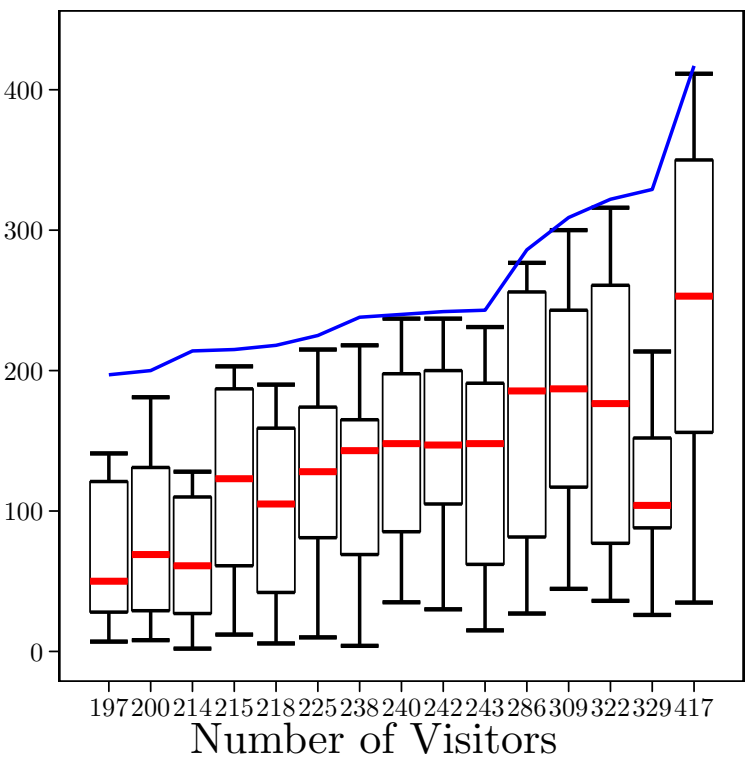

FIG. 17: (Color online) Results of spreading dynamics in the SG data: the figure shows panel boxplots of the final number of infected individuals in one day, versus the number of visitors in that day. The blue line represents the total number of daily visitors, giving an upper bound for the number of infected. The bottom and top of the rectangular boxes correspond to the $25^{t h}$ and $75^{t h}$ quantile of the distribution of infected individuals at the end of each day, and the red lines correspond to the median $\left(50^{t h}\right.$ quantile). The $5^{t h}$ and $95^{t h}$ are also shown (black horizontal lines).

final number of infected individuals, $N_{\text {inf }}$ to the number $N_{\text {sus }}$ of individuals who can be potentially reached through causal transmission paths starting at the seed. The distributions of this ratio is reported in Fig. 18. We observe that in the case of HT09 (left) almost all the potentially infected individuals will be infected by the end of the day, whereas the distribution of $N_{\mathrm{inf}} / N_{\text {sus }}$ is broader in the SG case (right). We notice that a static network description would inevitably lead to all individuals in the seed's CC catching the infection, a fact that can be a severe (and misleading) approximation of reality.

For each day the chosen seed generates a deterministic spreading process for which we can compute the cumulative number of infected individuals as a function of time, a quantity hereafter referred to as an incidence curve. Figure 19 shows the results for a selected day of the HT09 conference and for three different days of the SG data.

In the case of the HT09 conference, the earliest possible seeds are the conference organizers, but little happens until conference participants gather for the coffee break and/or meet up at the end of the first talk, between 10:00 

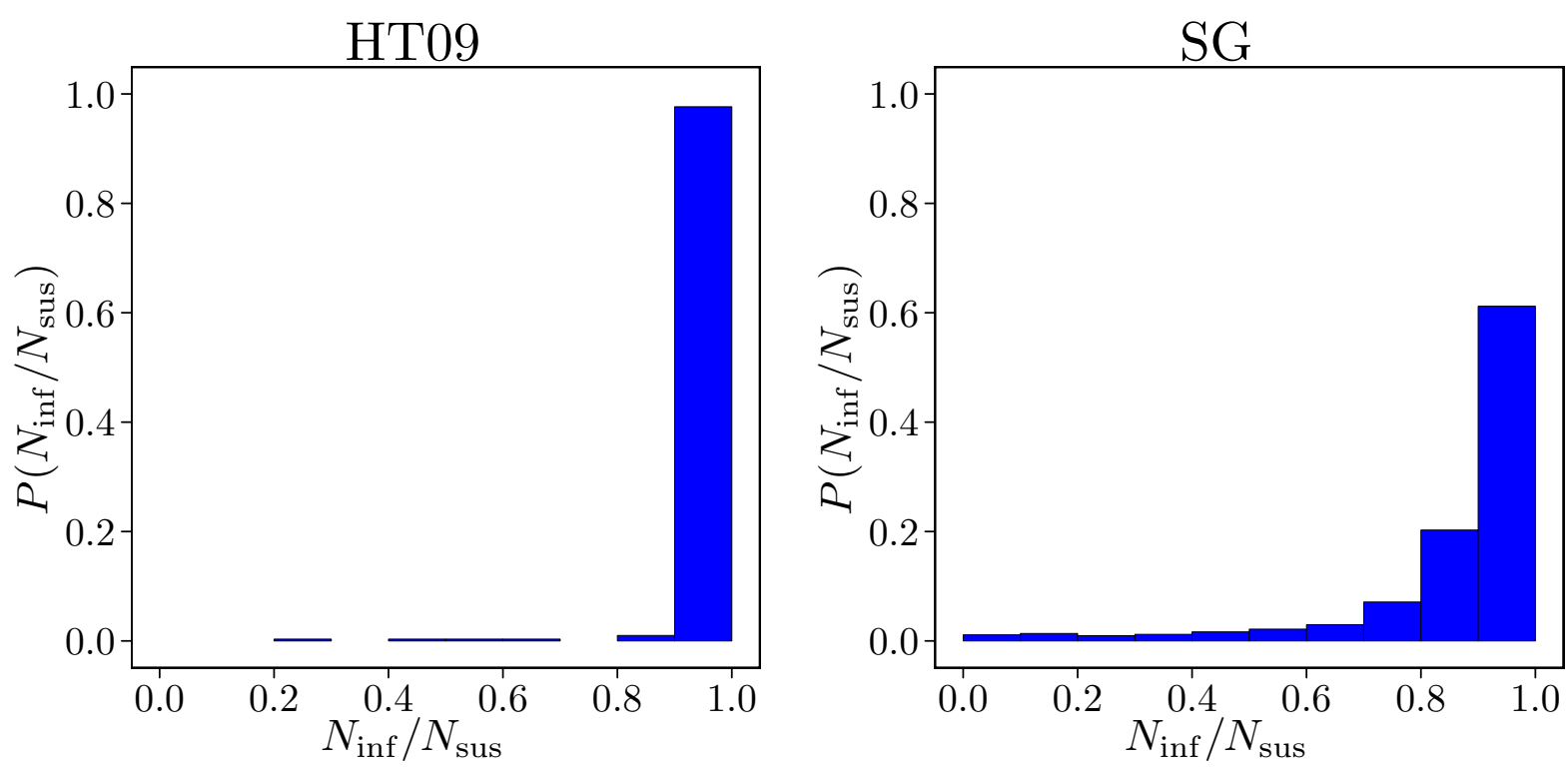

FIG. 18: Distribution of the ratio $N_{\text {inf }} / N_{\text {sus }}$ for the HT09 (left) and the SG (right) data, averaged over all potential seeds. $N_{\text {inf }}$ is the final number of infected individuals at the end of one day, while $N_{\text {sus }}$ is the number of individuals that could potentially be reached by a causal transmission path starting at the seed. $N_{\text {sus }}$ is given by the number of individuals visiting the premises in the same day, from the time the seed enters the premises, and belonging to the same CC as the seed.

and 11:00. A strong increase in the number of infected individuals is then observed, and a second strong increase occurs during the lunch break. Due to the concentration in time of transmission events, spreading processes reach very similar (and high) incidence levels after a few hours, regardless of the initial seed or its arriving time. Even processes started after 15:00 can reach about 80\% of the conference participants. Thus, the crucial point for the spreading process does not consist in knowing where and when the epidemic trajectory has started, but whether the seed or any other subsequently infected individual attend the coffee break or not.

A different picture is obtained in the SG case: First, in order to reach almost all participants the epidemics must spread on a globally connected network and start early (black curves for July $14^{\text {th }}$ data). Even in such a favorable setting for spreading, the incidence curves do not present sharp gradients, and later epidemics are unable to infect a large fraction of daily visitors. The incidence curves for May $19^{t h}$ and $20^{t h}$ of Fig. 19 show that different scenarios can also occur: due to the fragmented nature of the network, the final fraction of infected individuals can fluctuate greatly, and sharp increases of the incidence can be observed when dense groups such as those visible in Fig. 1 are reached.

\section{CONCLUSIONS}

In this paper we have shown that the analysis of time-resolved network data can unveil interesting properties of behavioral networks of face-to-face interaction between individuals. We considered data collected in two very different settings, representative of two types of social gatherings: the HT09 conference is a "closed" systems in which a group of individuals gathers and interacts in a repeated fashion, while the SG museum deployment is an "open" environment with a flux of individuals streaming through the premises.

We took advantage of the accurate time-resolved nature of our data sources to build dynamically evolving behavioral networks. We analyzed aggregated networks, constructed by aggregating the face-to-face interactions during time intervals of one day, and provided a comparison of their properties in both settings. We assessed the role of network dynamics on the outcome of dynamical processes such as spreading processes of informations or of an infectious agent.

Our analysis shows that the behavioral networks of individuals in conferences and in a museum setting exhibit both similarities and important differences. The topologies of the aggregated networks are widely different: the conference networks are rather dense small-worlds, while the SG networks have a larger diameter and are possibly made of several connected components - they do not form small-worlds, and their "elongated" shape can be put in relation with 

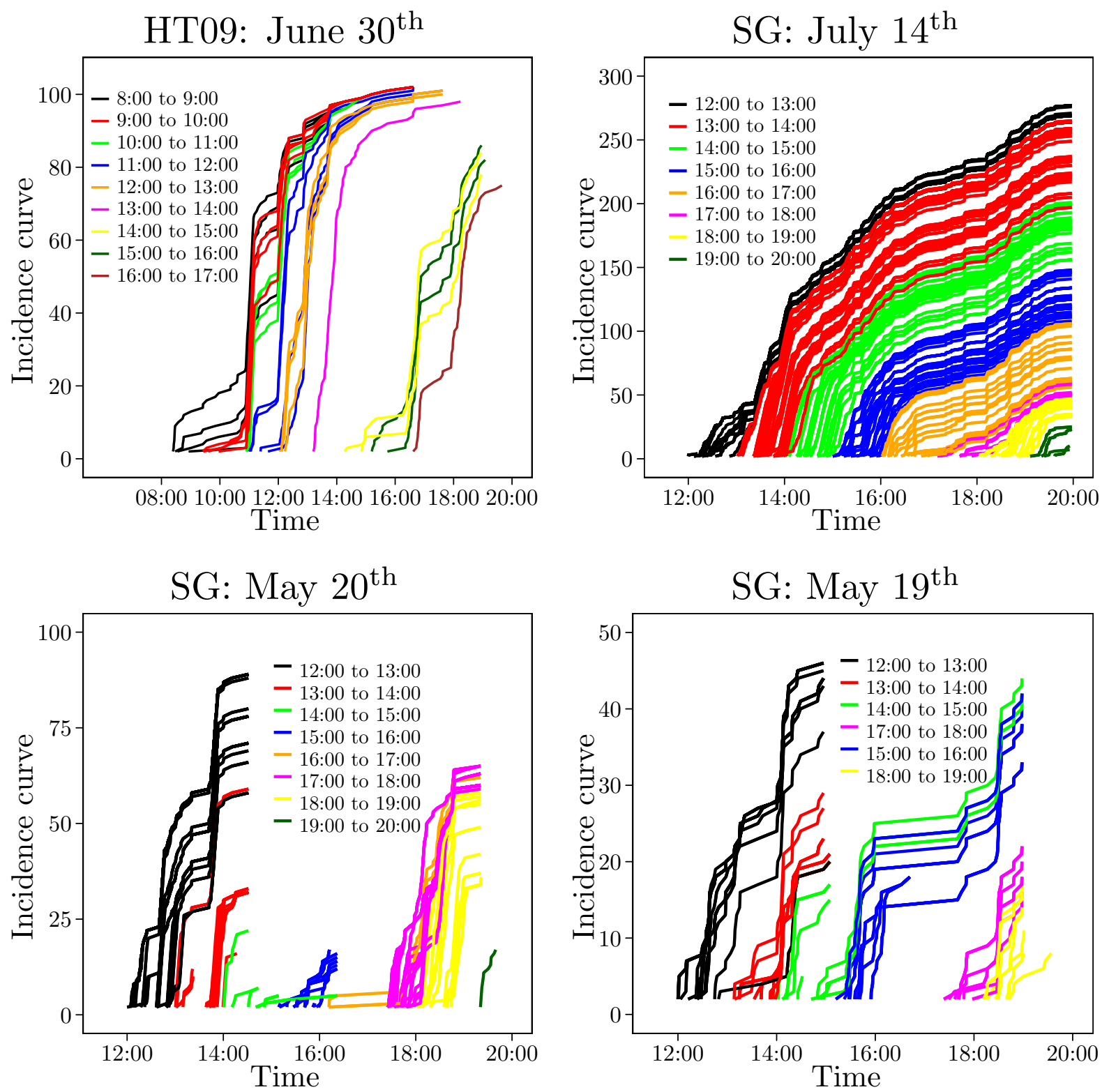

FIG. 19: (Color online) Incidence curves, giving the number of infected versus time for a spreading phenomenon simulated in the HT09 and SG data. Clockwise from top: HT09, June $30^{\text {th }}$ (aggregated network consisting of a single CC with $N_{1}=102$ individuals); SG network for July $14^{\text {th }}$ (one CC, $N_{1}=282$ individuals), May $19^{\text {th }}$ (two CCs, $N_{1}=N_{2}=49$ individuals) and May $20^{\text {th }}$ (two CCs, $N_{1}=N_{2}=89$ individuals). Each curve corresponds to a different seed, and is color-coded according to the starting time of the spreading.

the fact that individuals enter the premises at different times and remain there only for a limited amount of time. The networks' differences are also unveiled by a percolation analysis, which reveals how the SG aggregated networks can easily be dismantled by removing links that act as "bridges" between groups of individuals; on the contrary, aggregated networks at a conference are more "robust", even with respect to targeted link removal.

Interestingly, some important similarities are also observed: the degree distributions of aggregated networks, for example, are short-tailed in both cases. Moreover, despite the higher social activity at a conference, both the distribution of the contact event durations and the distribution of the total time spent in face-to-face interactions by two individuals are very similar.

The study of simple spreading processes unfolding on the dynamical networks of interaction between individuals allowed us to delve deeper into the time-resolved nature of our data. Comparison of the spreading dynamics on the time-dependent networks with the corresponding dynamics on the aggregated networks shows that the latter easily 
yields erroneous conclusions. In particular, our results highlight the strong impact of causality in the structure of transmission chains, that can differ significantly from those obtained on a static network. The temporal properties of the contacts are crucial in determining the spreading patterns and their properties. Studies about the role of the initial seed and its properties on the spreading patterns, or the determination of the most crucial nodes for propagation, can be misleading if only the static aggregated network is considered. In more realistic dynamics, the fastest path is typically not the shortest path of the aggregated network, and the role of causality is clearly visible in the analysis of the seed-to-last-infected paths.

Spreading phenomena unfold in very different ways in the two settings we investigated: at a conference, people interact repeatedly and with bursts of activity, so that transmission events also occur in a bursty fashion, and most individuals are reached at the end of the day; in a streaming situation, instead, the fraction of reached individuals can be very small due to either the lack of global connectivity or the late start of the spreading process. Detailed information on the temporal ordering of contacts is therefore crucial. We also note that in more realistic settings with non-deterministic spreading, information about the duration of contacts, and not only their temporal ordering, would also turn out to be very relevant and lead to an interesting interplay between the contact timescale and the propagation timescale [55. Future work will also address the issue of sampling effects: the fact that not all the conference attendees participated to the data collection may lead to an underestimation of spreading, since spreading paths between sampled attendees involving unobserved persons may have existed, but are not taken into account.

We close by stressing that as the data sources on person-to-person interactions become richer and ever more pervasive, the task of analyzing networks of interactions is unavoidably shifting away from statics towards dynamics, and a pressing need is building up for theoretical frameworks that can appropriately deal with streamed graph data and large scales. At the same time, we have shown that access to these data sources challenges a number of assumptions and poses new questions on how well-known dynamical processes unfold on dynamic graphs.

\section{Acknowledgments}

Data collections of the scale reported in this manuscript are only possible with the collaboration and support of many dedicated individuals. We gratefully thank the Science Gallery in Dublin for inspiring ideas and for hosting our deployment. Special thanks go to Michael John Gorman, Don Pohlman, Lynn Scarff, Derek Williams, and all the staff members and facilitators who helped to communicate the experiment and engage the public. We thank the organizers of the ACM Hypertext 2009 conference and acknowledge the help of Ezio Borzani, Vittoria Colizza, Daniela Paolotti, Corrado Gioannini and the staff members of the ISI Foundation, as well as the help of several Hypertext 2009 volunteers. We also thank Harith Alani, Martin Szomszor and Gianluca Correndo of the Live Social Semantics team. We warmly thank Bitmanufaktur and the OpenBeacon project, and acknowledge technical support from Milosch Meriac and Brita Meriac. We acknowledge stimulating discussions with Alessandro Vespignani and Vittoria Colizza. This study was partially supported by the FET-Open project DYNANETS (grant no. 233847) funded by the European Commission. Finally, we are grateful for the valuable feedback, the patience and the support of the tens of thousands of volunteers who participated in the deployments. 
[1] Special issue of Science on Complex networks and systems. Science 325 (2009) 357-504.

[2] S.N. Dorogovtsev, J.F.F. Mendes, Evolution of networks: From biological nets to the Internet and WWW, Oxford University Press, Oxford 2003.

[3] M.E.J. Newman, The structure and function of complex networks, SIAM Review 45 (2003) 167-256.

[4] R. Pastor-Satorras, A. Vespignani, Evolution and structure of the Internet: A statistical physics approach, Cambridge University Press, Cambridge, 2004.

[5] G. Caldarelli, Scale-Free Networks, Oxford University Press, Oxford, 2007.

[6] A. Barrat, M. Barthélemy, A. Vespignani, Dynamical processes on complex networks, Cambridge University Press, Cambridge, 2008.

[7] A. Wasserman, K. Faust, Social Network Analysis: Methods and applications, Cambridge University Press, Cambridge, 1994.

[8] D. Watts, Connections A twenty-first century science, Nature 445 (2007) 489.

[9] G. Chowell, J. M. Hyman, S. Eubank, C. Castillo-Chavez, Scaling laws for the movement of people between locations in a large city, Phys. Rev. E 68 (2003) 066102.

[10] A. De Montis, M. Barthélemy, A. Chessa, A. Vespignani, The structure of inter-urban traffic: a weighted network analysis, Environmental Planning Journal B 34 (2007) 905-924.

[11] D. Brockmann, L. Hufnagel, T. Geisel, The scaling laws of human travel, Nature 439 (2006) 462-465.

[12] A. Barrat, M. Barthélemy, R. Pastor-Satorras, A. Vespignani, The architecture of complex weighted networks, Proc. Natl. Acad. Sci. USA 101 (2004) 3747-3752.

[13] D. Balcan, V. Colizza, B. Gonçalves, H. Hu, J.J. Ramasco, A. Vespignani, Multiscale mobility networks and the spatial spreading of infectious diseases, Proc. Natl. Acad. Sci. USA 106 (2009) 21484-21489.

[14] M. C. González, C. A. Hidalgo, A.-L. Barabási, Understanding individual human mobility patterns, Nature 453 (2008) 779-782.

[15] C. Song, Z. Qu, N. Blumm, A.-L. Barabási, Limits of Predictability in Human Mobility, Science 327 (2010) $1018-1021$.

[16] J.-P. Eckmann, E. Moses, D. Sergi, Entropy of dialogues creates coherent structures in e-mail traffic, Proc. Natl. Acad. Sci. USA 101 (2004) 14333-14337.

[17] G. Kossinets, D. Watts, Empirical analysis of an evolving social network, Science 311 (2006) 88-90.

[18] S. Golder, D. Wilkinson, B. Huberman, Rhythms of social interaction: messaging within a massive online network, Communities and technologies 200\%: proceedings of the Third Communities and Technologies Conference, Michigan State University, 2007.

[19] J. Leskovec, E. Horvitz, Planetary-scale views on a large instant-messaging network, Proceeding of the 17th international conference on World Wide Web (2008) p 915-924, ACM New York, NY, USA.

[20] D. Rybski, S.V. Buldyrev, S. Havlin, F. Liljeros, H.A. Makse, Scaling laws of human interaction activity, Proc. Natl. Acad. Sci. USA 106 (2009) 12640-12645.

[21] R.D. Malmgren, D.B. Stouffer, A.S.L.O. Campanharo, L.A. Nunes Amaral, On Universality in Human Correspondence Activity, Science 325 (2009) 1696-1700.

[22] P. Hui, A. Chaintreau, J. Scott, R. Gass, J. Crowcroft, C. Diot, Pocket switched networks and human mobility in conference environments, Proceedings of the 2005 ACM SIGCOMM workshop on Delay-tolerant networking, Philadelphia, Pennsylvania, USA (2005) $244-251$.

[23] N. Eagle, A. Pentland, Reality mining: Sensing complex social systems, Personal and Ubiquitous Computing 10 (2006) 255-268.

[24] E. O’Neill, V. Kostakos, T. Kindberg, A. Fatah gen. Schieck, A. Penn, D. Stanton Fraser, T. Jones, Instrumenting the city: developing methods for observing and understanding the digital cityscape, Lecture Notes in Computer Science 4206 (2006) 315-332.

[25] A. Pentland, Honest Signals: how they shape our world, MIT Press, Cambridge MA, 2008.

[26] A. Clauset and N. Eagle, Persistence and periodicity in a dynamic proximity network, DIMACS Workshop on Computational Methods for Dynamic Interaction Networks, 2007.

[27] http://www.sociopatterns.org

[28] C. Cattuto, W. Van den Broeck, A. Barrat, V. Colizza, J.-F. Pinton, A. Vespignani, Dynamics of person-to-person interactions from distributed RFID sensor networks, PLoS ONE 5(7) (2010) e11596.

[29] H. Alani, M. Szomsor, C. Cattuto, W. Van den Broeck, G. Correndo, A. Barrat, Live Social Semantics, $8^{\text {th }}$ International Semantic Web Conference ISWC2009, Lecture Notes in Computer Science 5823 (2009) 698-714, http://dx.doi.org/10. 1007/978-3-642-04930-9_44

[30] W. Van den Broeck, C. Cattuto, A. Barrat, M. Szomsor, G. Correndo, H. Alani, The Live Social Semantics application: a platform for integrating face-to-face presence with on-line social networking, First International Workshop on Communication, Collaboration and Social Networking in Pervasive Computing Environments (PerCol 2010), Proceedings of the 8th Annual IEEE International Conference on Pervasive Computing and Communications, Mannheim, Germany (2010) 226-231.

[31] J.F. Padgett, C.K. Ansell, Robust action and the rise of the Medici. Am J Sociol 98 (1993) 1259-1319.

[32] M.J. Lubbers, J.L. Molina, J. Lerner, U. Brandes, J. Avila, C. McCarty, Longitudinal analysis of personal networks. The case of argentinean migrants in Spain, Social Networks, 32 (2010) 91-104. 
[33] http://www.sciencegallery.com/infectious

[34] http://www.ht2009.org/

[35] W. Willinger, R. Govindan, S. Jamin, V. Paxson, S. Shenker, Scaling phenomena in the Internet: Critically examining criticality. Proc. Natl. Acad. Sci. USA 99 (2002) 2573-2580.

[36] T. Petermann, P. De Los Rios, Exploration of scale-free networks: Do we measure the real exponents? Eur. Phys. J. B 38 (2004) 201-204.

[37] A. Clauset, C. Moore. Accuracy and scaling phenomena in Internet mapping, Phys. Rev. Lett. 94 (2005) 018701.

[38] L. Dall'Asta, I. Alvarez-Hamelin, A. Barrat, A. Vàzquez, A. Vespignani, Statistical theory of Internet exploration, Phys. Rev. E 71 (2005) 036135.

[39] S. Maslov, K. Sneppen, A. Zaliznyak, Detection of topological patterns in complex networks: correlation profile of the Internet, Physica A 333 (2004) 529-540.

[40] J.G. Oliveira, A.-L. Barabási, Darwin and Einstein correspondence patterns, Nature 437 (2005) 1251.

[41] J.-P. Onnela, J. Saramäki, J. Hyvonen, G. Szabó, M. Argollo de Menezes, K. Kaski, A.-L- Barabási, J. Kertész, Analysis of a large-scale weighted network of one-to-one human communication, New J. Phys 9 (2007) 179.

[42] R. Pastor-Satorras, A. Vespignani, Epidemic spreading in scale-free networks, Phys. Rev. Lett. 86 (2001) $3200-3203$.

[43] R.M. Anderson, R.M. May, Infectious Diseases of Humans: Dynamics and Control, Oxford Science Publications, Oxford, UK, 1992.

[44] R. Albert, H. Jeong, A.-L. Barabási, Error and attack tolerance of complex networks, Nature 406 (2000) $378-382$.

[45] R. Cohen, K. Erez, D. ben-Avraham, S. Havlin, Resilience of the Internet to random breakdowns, Phys. Rev. Lett. 85 (2000) 4626-4628.

[46] P. Holme, B. J. Kim, C. N. Yoon, S. K. Han, Attack vulnerability of complex networks, Phys. Rev. E 65 (2002) 056109.

[47] M. Girvan, M.E.J. Newman, Community structure in social and biological networks, Proc. Natl. Acad. Sci. USA 99 (2002) 7821-7826.

[48] F. Radicchi, C. Castellano, F. Cecconi, V. Loreto, D. Parisi, Defining and identifying communities in networks, Proc. Natl. Acad. Sci. USA 101 (2004) 2658-2663.

[49] L. Dall'Asta, A. Barrat, M. Barthélemy, A. Vespignani, Vulnerability of weighted networks, J. Stat. Mech. (2006) P04006.

[50] J.P. Onnela, J. Saramäki, J. Hyvönen, G. Szabó, D. Lazer, K. Kaski, J. Kertész, A.-L. Barabási, Structure and tie strengths in mobile communication networks, Proc. Natl. Acad. Sci. USA 104 (2007) 7332-7336.

[51] G. Kossinets, J. Kleinberg, D. Watts. The Structure of Information Pathways in a Social Communication Network, Proceedings of the $14^{t} h$ ACM SIGKDD International Conference on Knowledge Discovery and Data Mining (2008).

[52] E. Newman, W. Doran, N. Stokes, J. Carthy, J. Dunnion, Comparing Redundancy Removal Techniques for Multi-document Summarisation, Proceedings of STAIRS (2004) 223-228.

[53] C.J. van Rijsbergen, Information Retrieval, at http://www.dcs.gla.ac.uk/Keith/Preface.html.

[54] R. Baeza-Yates, B. Ribeiro-Neto, Modern Information Retrieval, ACM Press / Addison-Wesley, 1999.

[55] J. Stehlé, N. Voirin, A. Barrat, C. Cattuto, V. Colizza, L. Isella, C. Régis, J.-F. Pinton, N. Khanafer, W. Van den Broeck, P. Vanhems, Infectious disease spread on a data-driven dynamic contact network, submitted.

[56] G. Csardi and T. Nepusz, The igraph software package for complex network research, InterJournal Complex Systems (2006) 1695.

[57] As shown in Ref. 46, 49] and verified numerically (not shown) for the present case, a procedure that does not update the link ranking upon every link removal, based on the quantities (3) and (4), leads to sub-optimal results. The deviation from the updating strategy becomes apparent only when more than $20 \%$ of links have been removed since $O_{i j}$ and $\operatorname{sim}_{i j}$ deal with local quantities only. As a consequence, each link removal amounts to a local perturbation of the network, contrary to what happens with non-local quantities such as the betweenness centrality [46, 49]. 